\title{
Higgs CAT
}

\author{
Giampiero Passarino $0^{1,2, a}$ \\ ${ }^{1}$ Dipartimento di Fisica Teorica, Università di Torino, Turin, Italy \\ ${ }^{2}$ INFN, Sezione di Torino, Turin, Italy
}

Received: 7 April 2014 / Accepted: 17 April 2014 / Published online: 9 May 2014

(C) The Author(s) 2014. This article is published with open access at Springerlink.com

\begin{abstract}
Higgs Computed Axial Tomography, an excerpt. The Higgs boson lineshape (... and the devil hath power to assume a pleasing shape, Hamlet, Act II, scene 2) is analyzed for the $\mathrm{gg} \rightarrow \mathrm{ZZ}$ process, with special emphasis on the off-shell tail which shows up for large values of the Higgs virtuality. The effect of including background and interference is also discussed. The main focus of this work is on residual theoretical uncertainties, discussing how much-improved constraint on the Higgs intrinsic width can be revealed by an improved approach to analysis.
\end{abstract}

\section{Introduction}

Here I present a few personal recollections and observations on what is necessary in order to obtain the most accurate theoretical predictions outside the Higgs-like resonance region, given the present level of theoretical knowledge.

Somebody had an idea, somebody else gave it wings, a third group did the cut-and-count, and a fourth did a shape-based analysis. ${ }^{1}$ Ideas are like rabbits. You get a couple, learn how to handle them, and pretty soon you have a dozen.

In Ref. [1] the off-shell production cross section has been shown to be sizeable at high $\mathrm{ZZ}$-invariant mass in the gluon fusion production mode, with a ratio relative to the onpeak cross section of the order of $8 \%$ at a center-of-mass energy of $8 \mathrm{TeV}$. This ratio can be enhanced up to about $20 \%$ when a kinematical selection used to extract the signal in the resonant region is taken into account [2]. This arises from the vicinity of the on-shell $\mathrm{Z}$ pair production threshold, and is further enhanced at the on-shell top pair production threshold.

\footnotetext{
${ }^{1}$ Inspired by a friend.

a e-mail: giampiero@to.infn.it
}

In Ref. [3] the authors demonstrated that, with fewer assumptions and using events with pairs of $\mathrm{Z}$ particles, the high invariant mass tail can be used to constrain the Higgs width.

This note provides a more detailed description of the theoretical uncertainty associated with the camel-shaped and square-root-shaped tails of a light Higgs boson.

The outline of the paper is as follows: old and new ideas on measuring the Higgs boson intrinsic width are presented in Sect. 2, off-shell effects are discussed in Sect. 3, inclusion of the interference is analyzed in Sect. 3.4 with the introduction of different options for the corresponding theoretical uncertainty. Historical remarks are given in Sect. 4; in Sect. 4.2 improvements are introduced and critically analyzed.

\section{An old idea}

The problem of determining resonance parameters in $\mathrm{e}^{+} \mathrm{e}^{-}$ annihilation, including initial state radiative corrections and resolution corrections is an old one, see Ref. [4]. For the interested reader we recommend the original Refs. $[4,5]$ or the summary in Chap. 2 of Ref. [6].

\subsection{Higgs intrinsic width}

Is there anything we can say about what the intrinsic width of the light resonance is like? Ideas pass through three periods:

- It can't be done.

- It probably can be done, but it's not worth doing.

- I knew it was a good idea all along!

From the depths of my memory ...

Remark $X$ It can't be done: at LHC we reconstruct the invariant mass of the Higgs decay products, "easy" in case of $\gamma \gamma$ or 4 charged lepton final states. The mass resolution has a 
Gaussian core but non-Gaussian tails (e.g., due to calorimeter segmentation but also pile-up effects etc.). The accuracy in the mean of the mass peak can then approach that $1 . \%$ precision. Thus it could perhaps compare with the $\mathrm{W}$-mass extraction at LEP, based on some measured invariant mass distribution. Experimentalists would let the detector event simulation program do the folding of the theoretical invariant mass distribution, hoping that the MC catches most of the Gaussian and non-Gaussian resolution effects with the remainder being put into the systematic uncertainty. However, this would affect the width much more than the mass (mean of the distribution).

Remark $\boldsymbol{X}$ It's not worth doing. For the width of the Higgs things are thus much more difficult: For $M_{\mathrm{H}}<180 \mathrm{GeV}$ detector resolution dominates, so experimentally it will be very tough.

Let's review what we have learned in the meantime, highlighting new steps for Higgs precision physics:

- complete off-shell treatment of the Higgs signal

- signal-background interference

- residual theoretical uncertainty

\section{The wrath of the "heavy" Higgs}

You didn't want me to be real, I will contaminate your data, come and see if ye can swerve me

Let's see how this develops.

3.1 Higgs boson production and decay: the analytic structure

Remark $\checkmark$ I knew it was a good idea all along!

Before giving an unbiased description of production and decay of an Higgs boson we underline the general structure of any process containing a Higgs boson intermediate state. The corresponding amplitude is schematically given by

$A(s)=\frac{f(s)}{s-s_{\mathrm{H}}}+N(s)$,

where $N(s)$ denotes the part of the amplitude which is nonHiggs-resonant. Strictly speaking, signal (S) and background (B) should be defined as follows:

$$
\begin{gathered}
A(s)=S(s)+B(s), \quad S(s)=\frac{f\left(s_{\mathrm{H}}\right)}{s-s_{\mathrm{H}}}, \\
B(s)=\frac{f(s)-f\left(s_{\mathrm{H}}\right)}{s-s_{\mathrm{H}}}+N(s)
\end{gathered}
$$

Definition The Higgs complex pole (describing an unstable particle) is conventionally parametrized as

$s_{\mathrm{H}}=\mu_{\mathrm{H}}^{2}-i \mu_{\mathrm{H}} \gamma_{\mathrm{H}}$

As a first step we will show how to write $f(s)$ in a way such that pseudo-observables make their appearance $[7,8]$. Consider the process $i j \rightarrow \mathrm{H} \rightarrow \mathrm{F}$ where $i, j \in$ partons and $\mathrm{F}$ is a generic final state; the complete cross-section will be written as follows:

$$
\begin{aligned}
\sigma_{i j \rightarrow \mathrm{H} \rightarrow \mathrm{F}}(s)= & \frac{1}{2 s} \int d \Phi_{i j \rightarrow \mathrm{F}}\left[\sum_{s, c} \mid A_{\left.\left.i j \rightarrow \mathrm{H}\right|^{2}\right]}\right. \\
& \times \frac{1}{\left|s-s_{\mathrm{H}}\right|^{2}}\left[\sum_{s, c} \mid A_{\left.\left.\mathrm{H} \rightarrow \mathrm{F}\right|^{2}\right]}\right.
\end{aligned}
$$

where $\sum_{s, c}$ is over spin and colors (averaging on the initial state). Note that the background (e.g. gg $\rightarrow 4 f$ ) has not been included and, strictly speaking and for reasons of gauge invariance, one should consider only the residue of the Higgs-resonant amplitude at the complex pole, as described in Eq. (2). For gauge invariance the rule of thumb can be formulated by looking at Eq. (1): the only gauge invariant quantities are the location of the complex pole, its residue and the non-resonant part of the amplitude [ $B(s)$ of Eq. (2)]. For the moment we will argue that the dominant corrections are the QCD ones where we have no problem of gauge parameter dependence. If we decide to keep the Higgs boson off-shell also in the resonant part of the amplitude (interference signal/background remains unaddressed) then we can write

$$
\int d \Phi_{i j \rightarrow \mathrm{H}} \sum_{s, c}\left|A_{i j \rightarrow \mathrm{H}}\right|^{2}=s \bar{A}_{i j}(s) .
$$

For instance, we have

$\bar{A}_{\mathrm{gg}}(s)=\frac{\alpha_{\mathrm{s}}^{2}}{\pi^{2}} \frac{G_{\mathrm{F}} s}{288 \sqrt{2}}\left|\sum_{q} f\left(\tau_{q}\right)\right|^{2}\left(1+\delta_{\mathrm{QCD}}\right)$,

where $\tau_{q}=4 m_{q}^{2} / s, f\left(\tau_{q}\right)$ is defined in Eq. (3) of Ref. [9] and where $\delta_{\mathrm{QCD}}$ gives the QCD corrections to $\mathrm{gg} \rightarrow \mathrm{H}$ up to next-to-next-to-leading-order (NNLO) + next-to-leading logarithms (NLL) resummation. Furthermore, we define

$\Gamma_{\mathrm{H} \rightarrow \mathrm{F}}(s)=\frac{1}{2 \sqrt{s}} \int d \Phi_{\mathrm{H} \rightarrow \mathrm{F}} \sum_{s, c}\left|A_{\mathrm{H} \rightarrow \mathrm{F}}\right|^{2}$

which gives the partial decay width of a Higgs boson of virtuality $s$ into a final state F.

$\sigma_{i j \rightarrow \mathrm{H}}(s)=\frac{\bar{A}_{i j}(s)}{s}$

which gives the production cross-section of a Higgs boson of virtuality $s$. We can write the final result in terms of pseudoobservables 
Proposition 3.1 The familiar concept of on-shell production $\otimes$ branching ratio can be generalized to

$\sigma_{i j \rightarrow \mathrm{H} \rightarrow \mathrm{F}}(s)=\frac{1}{\pi} \sigma_{i j \rightarrow \mathrm{H}}(s) \frac{s^{2}}{\left|s-s_{\mathrm{H}}\right|^{2}} \frac{\Gamma_{\mathrm{H} \rightarrow \mathrm{F}}(s)}{\sqrt{s}}$

It is also convenient to rewrite the result as

$\sigma_{i j \rightarrow \mathrm{H} \rightarrow \mathrm{F}}(s)=\frac{1}{\pi} \sigma_{i j \rightarrow \mathrm{H}} \frac{s^{2}}{\left|s-s_{\mathrm{H}}\right|^{2}} \frac{\Gamma_{\mathrm{H}}^{\mathrm{tot}}}{\sqrt{s}} \mathrm{BR}(\mathrm{H} \rightarrow \mathrm{F})$

where we have introduced a sum over all final states,

$\Gamma_{\mathrm{H}}^{\mathrm{tot}}=\sum_{\mathrm{f} \in \mathrm{F}} \Gamma_{\mathrm{H} \rightarrow \mathrm{f}}$

Note that we have written the phase-space integral for $i\left(p_{1}\right)+$ $j\left(p_{2}\right) \rightarrow \mathrm{F}$ as

$$
\begin{aligned}
\int d \Phi_{i j \rightarrow \mathrm{F}}= & \int d^{4} k \delta^{4}\left(k-p_{1}-p_{2}\right) \\
& \times \int \prod_{f} d^{4} p_{f} \delta^{+}\left(p_{f}^{2}\right) \delta^{4}\left(k-\sum_{f} p_{f}\right)
\end{aligned}
$$

where we assume that all initial and final states (e.g. $\gamma \gamma, 4 \mathrm{f}$, etc.) are massless.

Why do we need pseudo-observables? Ideally experimenters (should) extract so-called realistic observables from raw data, e.g. $\sigma(\mathrm{pp} \rightarrow \gamma \gamma+\mathrm{X})$ and (should) present results in a form that can be useful for comparing them with theoretical predictions, i.e. the results should be transformed into pseudo-observables; during the deconvolution procedure one should also account for the interference background - signal; theorists (should) compute pseudo-observables using the best available technology and satisfying a list of demands from the self-consistency of the underlying theory.

Definition We define an off-shell production cross-section (for all channels) as follows:

$\sigma_{i j \rightarrow \text { all }}^{\text {prop }}=\frac{1}{\pi} \sigma_{i j \rightarrow \mathrm{H}} \frac{s^{2}}{\left|s-s_{\mathrm{H}}\right|^{2}} \frac{\Gamma_{\mathrm{H}}^{\mathrm{tot}}}{\sqrt{s}}$

When the cross-section $i j \rightarrow \mathrm{H}$ refers to an off-shell Higgs boson the choice of the QCD scales should be made according to the virtuality and not to a fixed value. Therefore, for the PDFs and $\sigma_{i j \rightarrow \mathrm{H}+\mathrm{X}}$ one should select $\mu_{\mathrm{F}}^{2}=\mu_{\mathrm{R}}^{2}=z s / 4$ ( $z s$ being the invariant mass of the detectable final state). Indeed, beyond lowest order (LO) one must not choose the invariant mass of the incoming partons for the renormalization and factorization scales, with the factor $1 / 2$ motivated by an improved convergence of fixed order expansion, but an infrared safe quantity fixed from the detectable final state, see Ref. [10]. The argument is based on minimization of the universal logarithms (DGLAP) and not the process-dependent ones.

\subsection{More on production cross-section}

We give the complete definition of the production crosssection; let us define $\zeta=z s, \kappa=v s$, and write

Definition $\sigma^{\text {prod }}$ is defined by the following equation:

$$
\begin{aligned}
\sigma^{\text {prod }} & =\sum_{i, j} \int_{i, j} \operatorname{PDF} \otimes \sigma_{i j \rightarrow \text { all }}^{\text {prod }} \\
& =\sum_{z_{0}} d z \int_{z}^{1} \frac{d v}{v} \mathscr{L}_{i j}(v) \sigma_{i j \rightarrow \text { all }}^{\text {prop }}\left(\zeta, \kappa, \mu_{\mathrm{R}}, \mu_{\mathrm{F}}\right)
\end{aligned}
$$

where $z_{0}$ is a lower bound on the invariant mass of the $\mathrm{H}$ decay products, the luminosity is defined by

$\mathscr{L}_{i j}(v)=\int_{v}^{1} \frac{d x}{x} f_{i}\left(x, \mu_{\mathrm{F}}\right) f_{j}\left(\frac{v}{x}, \mu_{\mathrm{F}}\right)$

where $f_{i}$ is a parton distribution function and

$$
\begin{aligned}
\sigma_{i j \rightarrow \text { all }}^{\text {prop }}\left(\zeta, \kappa, \mu_{\mathrm{R}}, \mu_{\mathrm{F}}\right)= & \frac{1}{\pi} \sigma_{i j \rightarrow \mathrm{H}+\mathrm{X}}\left(\zeta, \kappa, \mu_{\mathrm{R}}, \mu_{\mathrm{F}}\right) \\
& \times \frac{\zeta \kappa}{\left|\zeta-s_{\mathrm{H}}\right|^{2}} \frac{\Gamma_{\mathrm{H}}^{\mathrm{tot}}(\zeta)}{\sqrt{\zeta}}
\end{aligned}
$$

Therefore, $\sigma_{i j \rightarrow \mathrm{H}+\mathrm{X}}\left(\zeta, \kappa, \mu_{\mathrm{R}}\right)$ is the cross section for two partons of invariant mass $\kappa(z \leq v \leq 1)$ to produce a final state containing a $\mathrm{H}$ of virtuality $\zeta=z s$ plus jets $(\mathrm{X})$; it is made of several terms (see Ref. [9] for a definition of $\Delta \sigma$ ),

$$
\begin{aligned}
& \sum_{i j} \sigma_{i j \rightarrow \mathrm{H}+\mathrm{X}}\left(\zeta, \kappa, \mu_{\mathrm{R}}, \mu_{\mathrm{F}}\right)=\sigma_{\mathrm{gg} \rightarrow \mathrm{H}} \delta\left(1-\frac{z}{v}\right) \\
& +\frac{s}{\kappa}\left(\Delta \sigma_{\mathrm{gg} \rightarrow \mathrm{Hg}}+\Delta \sigma_{\mathrm{qg} \rightarrow \mathrm{Hq}}+\Delta \sigma_{\overline{\mathrm{q}} \mathrm{q} \rightarrow \mathrm{Hg}}+\mathrm{NNLO}\right)
\end{aligned}
$$

Remark As a technical remark the complete phase-space integral for the process $\hat{p}_{i}+\hat{p}_{j} \rightarrow p_{k}+\{f\}\left(\hat{p}_{i}=x_{i} p_{i}\right.$ etc.) is written as

$$
\begin{gathered}
\int d \Phi_{i j \rightarrow f}=\int d \Phi_{\mathrm{prod}} \int d \Phi_{\mathrm{dec}}=\int d^{4} p_{k} \delta^{+}\left(p_{k}^{2}\right) \\
\quad \times \prod_{l=1, n} d^{4} q_{l} \delta^{+}\left(q_{l}^{2}\right) \delta^{4}\left(\hat{p}_{i}+\hat{p}_{j}-p_{k}-\sum_{l} q_{l}\right) \\
=\int d^{4} k d^{4} Q \delta^{+}\left(p_{k}^{2}\right) \delta^{4}\left(\hat{p}_{i}+\hat{p}_{j}-p_{k}-Q\right) \\
\quad \times \int \prod_{l=1, n} d^{4} q_{l} \delta^{+}\left(q_{l}^{2}\right) \delta^{4}\left(Q-\sum_{l} q_{l}\right)
\end{gathered}
$$


where $\int d \Phi_{\mathrm{dec}}$ is the phase-space for the process $Q \rightarrow\{f\}$ and

$$
\begin{aligned}
\int & d \Phi_{\mathrm{prod}}=s \int d z \int d^{4} p_{k} d^{4} Q \delta^{+}\left(p_{k}^{2}\right) \delta\left(Q^{2}-\zeta\right) \theta\left(Q_{0}\right) \\
& \times \delta^{4}\left(\hat{p}_{i}+\hat{p}_{j}-p_{k}-Q\right) \\
= & s^{2} \int d z d v d \hat{t} \int d^{4} p_{k} d^{4} Q \delta^{+}\left(p_{k}^{2}\right) \delta\left(Q^{2}-\zeta\right) \theta\left(Q_{0}\right) \\
& \times \delta^{4}\left(\hat{p}_{i}+\hat{p}_{j}-p_{k}-Q\right) \\
& \times \delta\left(\left(\hat{p}_{i}+\hat{p}_{j}\right)^{2}-\kappa\right) \delta\left(\left(\hat{p}_{i}+Q\right)^{2}-\hat{t}\right)
\end{aligned}
$$

Equations (14) and (16) follow after folding with PDFs of argument $x_{i}$ and $x_{j}$, after using $x_{i}=x, x_{j}=v / x$ and after integration over $\hat{t}$. At NNLO there is an additional parton in the final state and five invariants are need to describe the partonic process, plus the $\mathrm{H}$ virtuality. However, one should remember that at NNLO use is made of the effective theory approximation where the Higgs-gluon interaction is described by a local operator.

\subsection{An idea that is not dangerous is unworthy} of being called an idea at all

Let us consider the case of a light Higgs boson; here, the common belief was that the product of on-shell production crosssection (say in gluon-gluon fusion) and branching ratios reproduces the correct result to great accuracy. The expectation is based on the well-known result [11] $\left(\Gamma_{\mathrm{H}} \ll M_{\mathrm{H}}\right)$

$$
\begin{aligned}
\Delta_{\mathrm{H}}= & \frac{1}{\left(s-M_{\mathrm{H}}^{2}\right)^{2}+\Gamma_{\mathrm{H}}^{2} M_{\mathrm{H}}^{2}}=\frac{\pi}{M_{\mathrm{H}} \Gamma_{\mathrm{H}}} \delta\left(s-M_{\mathrm{H}}^{2}\right) \\
& +\mathrm{PV}\left[\frac{1}{\left(s-M_{\mathrm{H}}^{2}\right)^{2}}\right]
\end{aligned}
$$

where PV denotes the principal value (understood as a distribution). Furthermore $s$ is the Higgs virtuality and $M_{\mathrm{H}}$ and $\Gamma_{\mathrm{H}}$ should be understood as $M_{\mathrm{H}}=\mu_{\mathrm{H}}$ and $\Gamma_{\mathrm{H}}=\gamma_{\mathrm{H}}$ and not as the corresponding on-shell values. In more simple terms, the first term in Eq. (20) puts you on-shell and the second one gives you the off-shell tail. More details are given in Appendix A.

Remark $\Delta_{\mathrm{H}}$ is the Higgs propagator, there is no space for anything else in QFT (e.g. Breit-Wigner distributions). For a comparison of Breit-Wigner and Complex Pole distributed cross sections at $\mu_{\mathrm{H}}=125.6 \mathrm{GeV}$ see Figs. 1 and 2 .

A more familiar representation of the propagator can be written as follows:

Definition with the parametrization of Eq. (3) we perform the well-known transformation

$\bar{M}_{\mathrm{H}}^{2}=\mu_{\mathrm{H}}^{2}+\gamma_{\mathrm{H}}^{2} \quad \mu_{\mathrm{H}} \bar{\Gamma}_{\mathrm{H}}=\bar{M}_{\mathrm{H}} \gamma_{\mathrm{H}}$

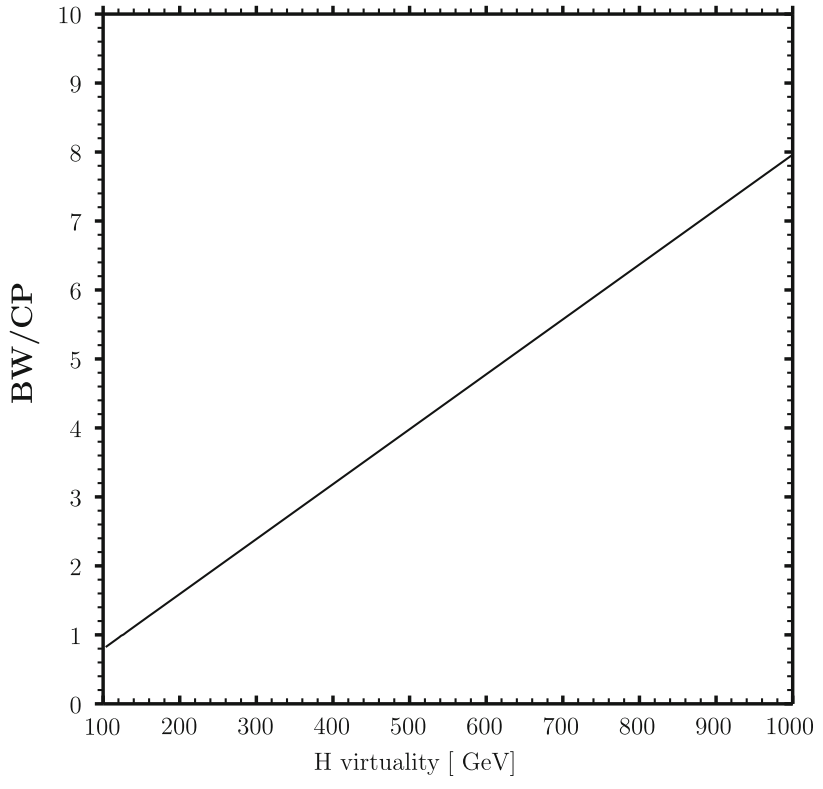

Fig. 1 Ratio of Breit-Wigner and Complex Pole distributed cross sections at $\mu_{\mathrm{H}}=125.6 \mathrm{GeV}$

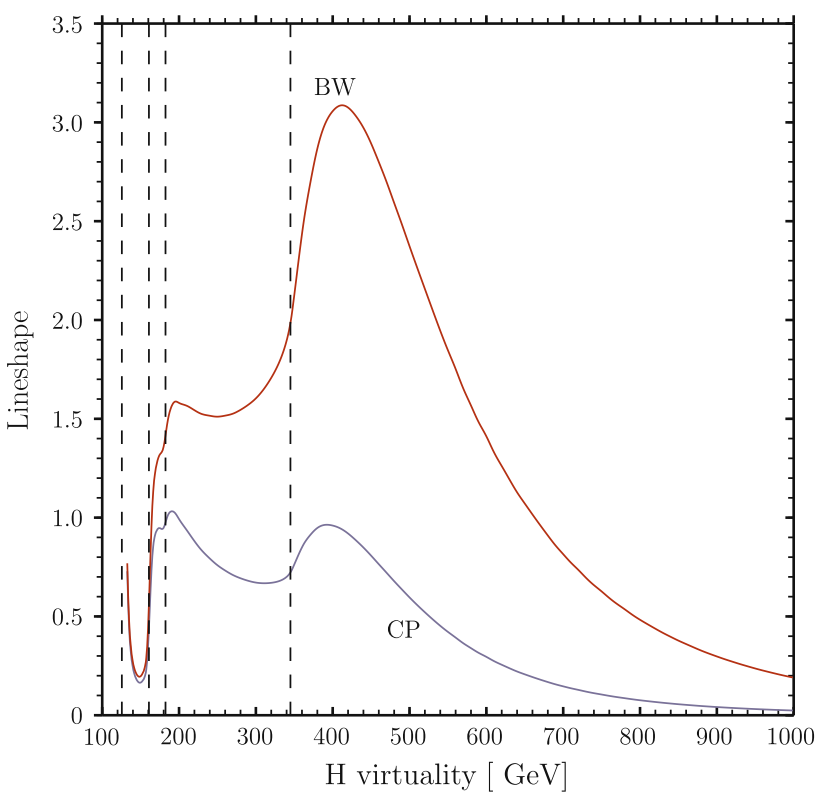

Fig. 2 Breit-Wigner and Complex Pole distributed lineshapes at $\mu_{\mathrm{H}}=$ $125.6 \mathrm{GeV}$

A remarkable identity follows (defining the Bar-scheme):

$\frac{1}{s-s_{\mathrm{H}}}=\left(1+i \frac{\bar{\Gamma}_{\mathrm{H}}}{\bar{M}_{\mathrm{H}}}\right)\left(s-\bar{M}_{\mathrm{H}}^{2}+i \frac{\bar{\Gamma}_{\mathrm{H}}}{\bar{M}_{\mathrm{H}}} s\right)^{-1}$

showing that the Bar-scheme is equivalent to introducing a running width in the propagator with parameters that are not the on-shell ones. Special attention goes to the numerator in Eq. (22) which is essential in providing the right asymptotic 
behavior when $s \rightarrow \infty$, as needed for cancellations with contact terms in VV scattering.

The natural question is: to which level of accuracy does the ZWA [delta-term only in Eq. (20)] approximate the full off-shell result given that at $\mu_{\mathrm{H}}=125 \mathrm{GeV}$ the on-shell width is only $4.03 \mathrm{MeV}$ ? For definiteness we will consider $i j \rightarrow \mathrm{H} \rightarrow \mathrm{ZZ} \rightarrow 41$. When searching the Higgs boson around $125 \mathrm{GeV}$ one should not care about the region $M_{\mathrm{ZZ}}>$ $2 M_{Z}$ but, due to limited statistics, theory predictions for the normalization in $\overline{\mathrm{q}}-\mathrm{q}-\mathrm{gg} \rightarrow \mathrm{ZZ}$ are used over the entire spectrum in the $\mathrm{ZZ}$ invariant mass.

Therefore, the question is not to dispute that off-shell effects are depressed by a factor $\gamma_{\mathrm{H}} / \mu_{\mathrm{H}}$ but to move away from the peak and look at the behavior of the invariant mass distribution, no matter how small it is compared to the peak; is it really decreasing with $M_{\mathrm{ZZ}}$ ? Is there a plateau? For how long? How does that affect the total cross-section if no cut is made?

Let us consider the signal, in the complex-pole scheme:

$$
\begin{aligned}
\sigma_{\mathrm{gg} \rightarrow \mathrm{ZZ}}(\mathrm{S}) & =\sigma_{\mathrm{gg} \rightarrow \mathrm{H} \rightarrow \mathrm{ZZ}}\left(M_{\mathrm{ZZ}}^{2}\right) \\
& =\frac{1}{\pi} \sigma_{\mathrm{gg} \rightarrow \mathrm{H}} \frac{M_{\mathrm{ZZ}}^{4}}{\left|M_{\mathrm{ZZ}}^{2}-s_{\mathrm{H}}\right|^{2}} \frac{\Gamma_{\mathrm{H} \rightarrow \mathrm{ZZ}}\left(M_{\mathrm{Z}}\right)}{M_{\mathrm{ZZ}}}
\end{aligned}
$$

where $s_{\mathrm{H}}$ is the Higgs complex pole, given in Eq. (3). Away (but not too far away) from the narrow peak the propagator and the off-shell $\mathrm{H}$ width behave like

$\Delta_{\mathrm{H}} \approx \frac{1}{\left(M_{\mathrm{ZZ}}^{2}-\mu_{\mathrm{H}}^{2}\right)^{2}}, \quad \frac{\Gamma_{\mathrm{H} \rightarrow \mathrm{ZZ}}\left(M_{\mathrm{Z}}\right)}{M_{\mathrm{ZZ}}} \sim G_{\mathrm{F}} M_{\mathrm{ZZ}}^{2}$

above threshold with a sharp increase just below it (it goes from $1.62 \times 10^{-2} \mathrm{GeV}$ at $175 \mathrm{GeV}$ to $1.25 \times 10^{-1} \mathrm{GeV}$ at $185 \mathrm{GeV})$.

Our result for the VV $(\mathrm{V}=\mathrm{W} / \mathrm{Z})$ invariant mass distribution is shown in Fig. 3: after the peak the distribution is falling down until the effects of the VV -thresholds become effective with a visible increase followed by a plateau, by another jump at the $\overline{\mathrm{t}}-\mathrm{t}$-threshold. Finally the signal distribution starts again to decrease, almost linearly.

What is the net effect on the total cross-section? We show it in Table 1 where the contribution above the $\mathrm{ZZ}$-threshold amounts to $7.6 \%$. The presence of the effect does not depend on the propagator function used (Breit-Wigner or complexpole propagator). The size of the effect is related to the distribution function. In Table 2 we present the invariant mass distribution integrated bin-by-bin.

If we take the ZWA value for the production cross-section at $8 \mathrm{TeV}$ and for $\mu_{\mathrm{H}}=125 \mathrm{GeV}(19.146 \mathrm{pb})$ and use the branching ratio into $\mathrm{ZZ}$ of $2.67 \times 10^{-2}$ we obtain a ZWA result of $0.5203 \mathrm{pb}$ with a $5 \%$ difference w.r.t. the off-shell result, fully compatible with the $7.6 \%$ effect coming form the high-energy side of the resonance.

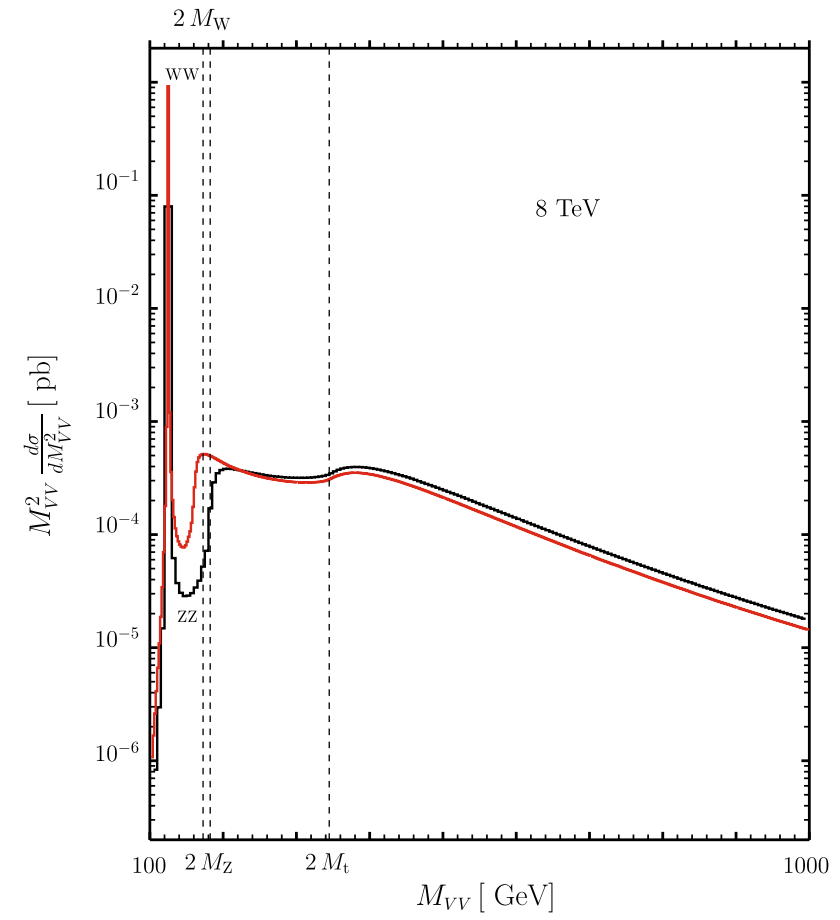

Fig. 3 The NNLO VV invariant mass distribution in gg $\rightarrow$ VV for $\mu_{\mathrm{H}}=125 \mathrm{GeV}$

Table 1 Total cross-section in $\mathrm{gg} \rightarrow \mathrm{H} \rightarrow \mathrm{ZZ}$ and in $\mathrm{gg} \rightarrow \mathrm{H} \rightarrow$ all; the part of the cross-section for $M_{\mathrm{ZZ}}>2 M_{\mathrm{Z}}$ is explicitly shown. $R[\%]$ is the ratio between the number of events with $M_{\mathrm{ZZ}}>2 M_{\mathrm{Z}}$ and the total number of events

\begin{tabular}{lcll}
\hline & Tot $(\mathrm{pb})$ & $M_{\mathrm{ZZ}}>2 M_{\mathrm{Z}}(\mathrm{pb})$ & $\mathrm{R}(\%)$ \\
\hline $\mathrm{gg} \rightarrow \mathrm{H} \rightarrow$ all & 19.146 & 0.1525 & 0.8 \\
$\mathrm{gg} \rightarrow \mathrm{H} \rightarrow \mathrm{ZZ}$ & 0.5462 & 0.0416 & 7.6 \\
\hline
\end{tabular}

Always from Table 1 we see that the effect is much less evident if we sum over all final states with a net effect of only $0.8 \%$ (the decay is $\bar{b}-\mathrm{b}$ dominated).

Of course, the signal per se is not a physical observable and one should always include background and interference. In Fig. 4 we show the complete LO result. Numbers are shown with a cut of $0.25 \mathrm{M}_{\mathrm{ZZ}}$ on $p_{\mathrm{T}}^{\mathrm{Z}}$. The large destructive effects of the interference wash out the peculiar structure of the signal distribution. If one includes the region $M_{\mathrm{ZZ}}>2 M_{\mathrm{Z}}$ in the analysis then the conclusion is: interference effects are relevant also for the low-mass region.

It is worth noting again that the whole effect on the signal has nothing to do with $\gamma_{\mathrm{H}} / \mu_{\mathrm{H}}$ effects; above the ZZ threshold the distribution is higher than expected (although tiny w.r.t. the narrow peak) and stays approximately constant till the $\overline{\mathrm{t}}-\mathrm{t}$-threshold after which we observe an almost linear decrease. This is why the total cross-section is affected (in a VV final state) at the 5\% level. 
Table 2 Bin-by-bin cross-section in $\mathrm{gg} \rightarrow \mathrm{H} \rightarrow \mathrm{ZZ}$. First row gives the bin in $\mathrm{GeV}$, second row gives the cross-section in pb

\begin{tabular}{llllllll}
\hline $100-125$ & $125-150$ & $150-175$ & $175-200$ & $200-225$ & $225-250$ & $250-275$ & $275-300$ \\
0.252 & 0.252 & $0.195 \times 10^{-3}$ & $0.177 \times 10^{-2}$ & $0.278 \times 10^{-2}$ & $0.258 \times 10^{-2}$ & $0.240 \times 10^{-2}$ & $0.230 \times 10^{-2}$ \\
\hline
\end{tabular}

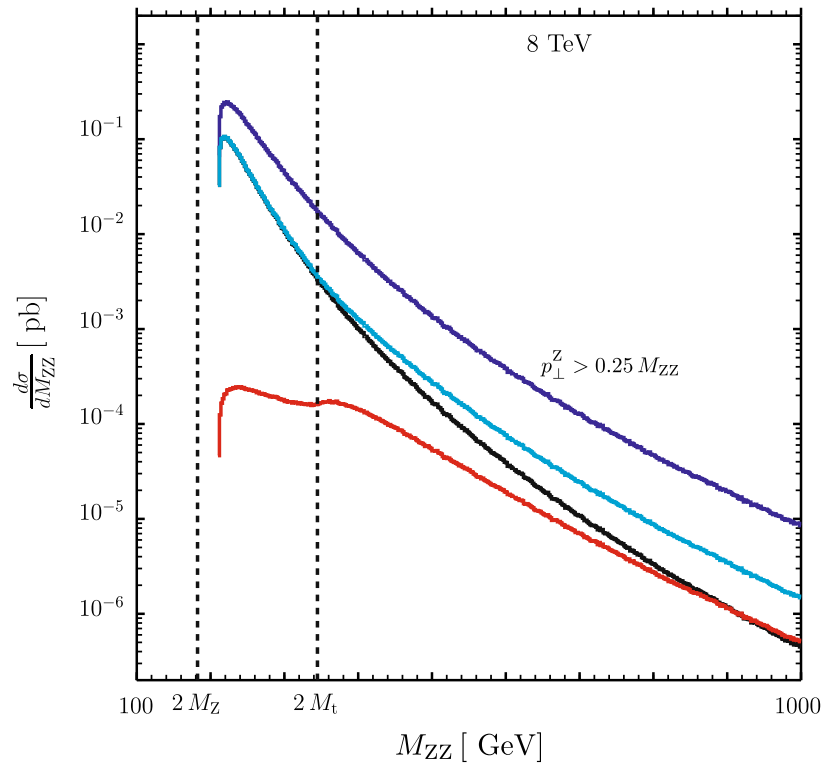

Fig. 4 The LO ZZ invariant mass distribution $\mathrm{gg} \rightarrow \mathrm{ZZ}$ for $\mu_{\mathrm{H}}=$ $125 \mathrm{GeV}$. The black line is the total, the red line gives the signal while the cyan line gives signal plus background; the blue line includes the $q \bar{q} \rightarrow \mathrm{ZZ}$ contribution

\subsection{When the going gets tough, interference gets going}

The higher-order correction in gluon-gluon fusion have shown a huge $\mathrm{K}$-factor

$\mathrm{K}=\frac{\sigma_{\text {prod }}^{\mathrm{NNO}}}{\sigma_{\text {prod }}^{\mathrm{LO}}}, \quad \sigma_{\text {prod }}=\sigma_{\mathrm{gg} \rightarrow \mathrm{H}}$.

\subsubsection{The zero-knowledge scenario}

A potential worry is: should we simply use the full $\mathrm{LO}$ calculation or should we try to effectively include the large (factor two) K -factor to have effective NNLO observables? There are different opinions since interference effects may be as large or larger than NNLO corrections to the signal. Therefore, it is important to quantify both effects. We examine first the scenario where zero knowledge is assumed on the $\mathrm{K}$-factor for the background. So far, two options have been introduced to account for the increase in the signal. Let us consider any distribution $\mathrm{D}$ (for definiteness we will consider $i j \rightarrow \mathrm{H} \rightarrow \mathrm{ZZ} \rightarrow 4 \mathrm{l})$, i.e.

$D=\frac{d \sigma}{d M_{\mathrm{ZZ}}^{2}}$ or $\frac{d \sigma}{d p_{\mathrm{T}}^{\mathrm{Z}}}$ etc. where $M_{\mathrm{ZZ}}$ is the invariant mass of the $\mathrm{ZZ}$-pair and $p_{\mathrm{T}}^{\mathrm{Z}}$ is the transverse momentum. Two possible options are:

Definition The additive option is defined by the following relation

$\mathrm{D}_{\mathrm{eff}}^{\mathrm{NNLO}}=\mathrm{D}^{\mathrm{NNLO}}(\mathrm{S})+\mathrm{D}^{\mathrm{LO}}(\mathrm{I})+\mathrm{D}^{\mathrm{LO}}(\mathrm{B})$

Definition The multiplicative [12] (M) or completely multiplicative $(\overline{\mathrm{M}})$ option is defined by the following relation:

$$
\begin{gathered}
\mathrm{D}_{\mathrm{eff}}^{\mathrm{NNLO}}(\mathrm{M})=\mathrm{K}_{\mathrm{D}}\left[\mathrm{D}^{\mathrm{LO}}(\mathrm{S})+\mathrm{D}^{\mathrm{LO}}(\mathrm{I})\right]+\mathrm{D}^{\mathrm{LO}}(\mathrm{B}), \\
\mathrm{D}_{\mathrm{eff}}^{\mathrm{NNO}}(\overline{\mathrm{M}})=\mathrm{K}_{\mathrm{D}} \mathrm{D}^{\mathrm{LO}}, \quad \mathrm{K}_{\mathrm{D}}=\frac{\mathrm{D}^{\mathrm{NNLO}}(\mathrm{S})}{\mathrm{D}^{\mathrm{LO}}(\mathrm{S})}
\end{gathered}
$$

where $\mathrm{K}_{\mathrm{D}}$ is the differential $\mathrm{K}$-factor for the distribution. The $\overline{\mathrm{M}}$ option is only relevant for background subtraction and it is closer to the central value described in Sect. 4.2.1.

In both cases the NNLO corrections include the NLO electroweak (EW) part, for production [13] and decay. The EW NLO corrections for $\mathrm{H} \rightarrow \mathrm{WW} / \mathrm{ZZ} \rightarrow 4 \mathrm{f}$ can reach a $15 \%$ in the high part of the tail. It is worth noting that the differential $\mathrm{K}$-factor for the $\mathrm{ZZ}$-invariant mass distribution is a slowly increasing function of $M_{\mathrm{ZZ}}$ after $M_{\mathrm{ZZ}}=2 M_{\mathrm{t}}$, going (e.g. for $\mu_{\mathrm{H}}=125.6 \mathrm{GeV}$ ) from 1.98 at $M_{\mathrm{ZZ}}=2 M_{\mathrm{t}}$ to 2.11 at $M_{\mathrm{ZZ}}=1 \mathrm{GeV}$.

The two options, as well as intermediate ones, suffer from an obvious problem: they are spoiling the unitarity cancellation between signal and background for $M_{\mathrm{ZZ}} \rightarrow \infty$. Therefore, our partial conclusion is that any option showing an early onset of unitarity violation should not be used for too high values of the $\mathrm{ZZ}$-invariant mass.

Therefore, our first prescription in proposing an effective higher-order interference will be to limit the risk of overestimation of the signal by applying the recipe only in some restricted interval of the ZZ -invariant mass. This is especially true for high values of $\mu_{\mathrm{H}}$ where the off-shell effect is large. Explicit calculations show that the multiplicative option is better suited for regions with destructive interference while the additive option can be used in regions where the effect of the interference is positive, i.e. we still miss higher orders from the background amplitude but do not spoil cancellations between signal and background.

Actually, there is an intermediate options that is based on the following observation: higher-order corrections to the signal are made of several terms, see Eq. (14): the partonic cross-section is defined by 


$$
\begin{aligned}
& \sum_{i j} \sigma_{i j \rightarrow \mathrm{H}+\mathrm{X}}\left(\zeta, \kappa, \mu_{\mathrm{R}}, \mu_{\mathrm{F}}\right)=\sigma_{\mathrm{gg} \rightarrow \mathrm{H}} \delta\left(1-\frac{z}{v}\right) \\
& +\frac{s}{\kappa}\left(\Delta \sigma_{\mathrm{gg} \rightarrow \mathrm{Hg}}+\Delta \sigma_{\mathrm{qg} \rightarrow \mathrm{Hq}}+\Delta \sigma_{\overline{\mathrm{q}} \rightarrow \mathrm{Hg}}+\mathrm{NNLO}\right)
\end{aligned}
$$

From this point of view it seems more convenient to define

$\mathrm{K}_{\mathrm{D}}=\mathrm{K}_{\mathrm{D}}^{\mathrm{gg}}+\mathrm{K}_{\mathrm{D}}^{\mathrm{rest}}, \quad \mathrm{K}_{\mathrm{D}}^{\mathrm{gg}}=\frac{\mathrm{D}^{\mathrm{NNLO}}(\mathrm{gg} \rightarrow \mathrm{H}(\mathrm{g}) \rightarrow \mathrm{ZZ}(\mathrm{g}))}{\mathrm{D}^{\mathrm{LO}}(\mathrm{gg} \rightarrow \mathrm{H} \rightarrow \mathrm{ZZ})}$

and to introduce a third option

Definition The intermediate option is given by the following relation:

$\mathrm{D}_{\mathrm{eff}}^{\mathrm{NNLO}}=\mathrm{K}_{\mathrm{D}} \mathrm{D}^{\mathrm{LO}}(\mathrm{S})+\left(\mathrm{K}_{\mathrm{D}}^{\mathrm{gg}}\right)^{1 / 2} \mathrm{D}^{\mathrm{LO}}(\mathrm{I})+\mathrm{D}^{\mathrm{LO}}(\mathrm{B})$

which, in our opinion, better simulates the inclusion of $\mathrm{K}$ factors at the level of amplitudes in the zero knowledge scenario (where we are still missing corrections to the continuum amplitude).

\section{There is no free lunch}

Summary of (Higgs precision physics) milestones without sweeping under the rug the following issues:

- moving forward, beyond ZWA (see Ref. [1]) don't try fixing something that is already broken in the first place.

- Unstable particles require complex-pole-scheme (see Ref. [14]).

- Off-shell + Interferences + uncertainty in VV production (see Ref. [15]).

- See also Interference in di-photon channel, see Refs. [16, 17] and Refs. [18-20].

The so-called area method [4] is not so useless, even for a light Higgs boson. One can use a measurement of the off-shell region to constrain the couplings of the Higgs boson. Using a simple cut-and-count method and one scaling parameter (see Eq. (32) in Sect. 4.1), existing LHC data should bound the width at the level of 25-45 times the Standard Model expectation $[3,21]$.

Remark Chronology and Historical background one cannot influence developments beyond telling his side of the story. The judgement about originality, importance, impact etc. is of course up to others

- Constraining the Higgs boson intrinsic width has been discussed during several LHC HXSWG meetings (G. Passarino, LHC HXSWG epistolar exchange, e.g. 10/25/10 with CMS "Are you referring to measuring the width according to the area method you discuss in your book [6]? That would be interesting to apply if possible").

- N. Kauer was the first person who created a plot clearly showing the enhanced Higgs tail. It was shown at the 6th LHC HXSWG meeting. ${ }^{2}$

- N. Kauer and G. Passarino (arXiv:1206.4803 [hep-ph]) confirmed the tail and provided an explanation for it, starting a detailed phenomenological study, see Ref. [1] and also Refs. [2,22].

- Higgs interferometry has been discussed at length in the LHC HXSWG (epistolar exchange, e.g. on 05/17/13 “... the interference effects could be used to constrain BSM Higgs via indirect Higgs width measurement ... there are large visible effects ${ }^{3}$ ). For a comprehensive presentation, see D. de Florian talk at "Higgs Couplings 2013". 4

- Dixon and Li [19], followed by F. Caola and K. Melnikov (arXiv:1307.4935 [hep-ph]) introduced the notion of $\infty$-degenerate solutions for the Higgs couplings to SM particles, observed that the enhanced tail, discussed and explained in arXiv:1206.4803 [hep-ph], is obviously $\gamma_{\mathrm{H}}$ independent and that this could be exploited to constrain the Higgs width model-independently if there's experimental sensitivity to the off-peak Higgs signal [3]. Once you have a model for increasing the width beyond the SM value, Ref. [3] turns the observation of Ref. [1] into a bound on the Higgs width, within the given scenario of degeneracy.

- J. M. Campbell, R. K. Ellis and C. Williams (arXiv:1311.3589 [hep-ph]) investigated the power of using a matrix element method (MEM) to construct a kinematic discriminant to sharpen the constraint [21] (with foreseeable extensions in MEM@NLO [23]). MEM-based analysis has been the first to describe a method for suppressing $\overline{\mathrm{q}}-\mathrm{q}$ background; the importance of his work cannot be overestimated. Complementary results from $\mathrm{H} \rightarrow \mathrm{WW}$ in the high transverse mass region are shown in Ref. [24].

The MEM-based analysis for separation of the $\mathrm{gg} \rightarrow \mathrm{ZZ}$ and $\overline{\mathrm{q}} \mathrm{q} \rightarrow \mathrm{ZZ}$ processes, including signal and background interference within the $\mathrm{gg} \rightarrow \mathrm{ZZ}$ process, has been suggested and implemented within the MELA framework on CMS [25] (http://www.pha.jhu.edu/spin/).

- This note provides a more detailed description of the theoretical uncertainty associated with the camel-shaped and square-root-shaped tails of a light Higgs boson.

\footnotetext{
2 https://indico.cern.ch/conferenceDisplay.py?ovw=True\&confId $=18$ 2952.

${ }^{3}$ See R. Tanaka talk at http://indico.cern.ch/conferenceTimeTable.py? confId=202554\#all.detailed.

${ }^{4}$ https://indico.cern.ch/contributionListDisplay.py?confId=253774.
} 


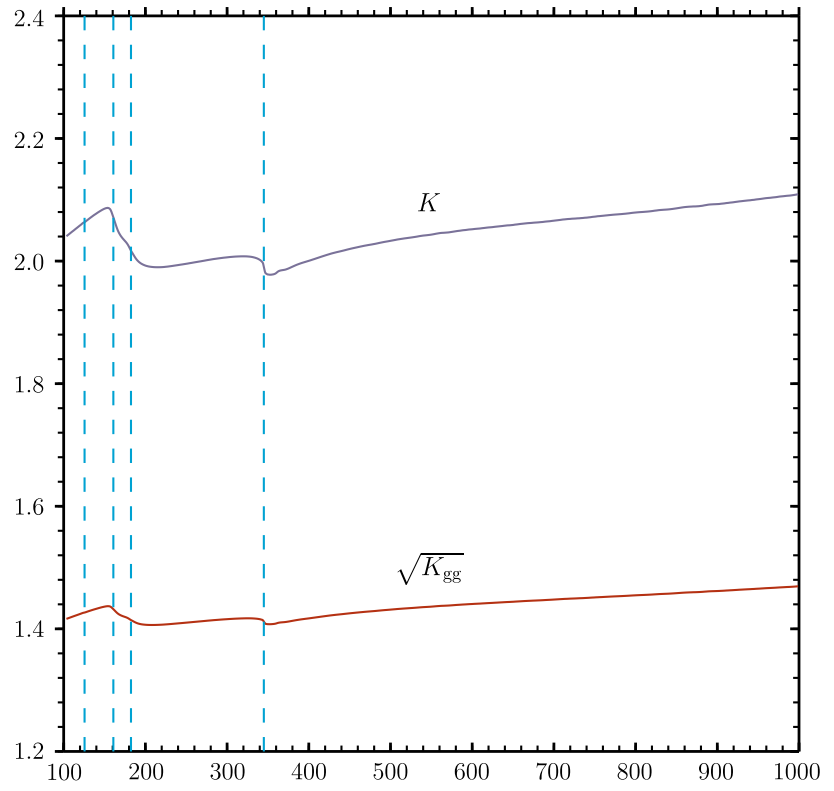

Fig. 5 Differential $K$-factors in Higgs production for $\mu_{\mathrm{H}}=$ $125.6 \mathrm{GeV}$

- A similar analysis, performed for the exclusion of a heavy SM Higgs boson, can be found in Ref. [15] and in Ref. [12] with improvements suggested in Ref. [26].

\subsection{How to use an LO MC?}

The MCs used in the analysis are based on LO calculations, some of them include $\mathrm{K}$-factors for the production but all of them have decay and interference implemented at LO. The adopted solution is external "re-weighting" (i.e. re-weighting with results from some analytical code), although rescaling exclusive distributions (e.g. in the final state leptons) with inclusive $\mathrm{K}$-factors is something that should not be done, it requires (at least) a $1-1$ correspondence between the two lowest orders.

An example of $\mathrm{K}$-factors that can be used to include interference in the zero-knowledge scenario is given in Fig. 5. For a more general discussion on re-weighting see Ref. [27].

Most of the studies performed so far are for the exclusion of a heavy SM Higgs boson ${ }^{5}$ and, from that experience, we can derive that It Takes A Fool To Remain Sane:

\section{A list of comments and/or problems}

- LO decay is not state-of-art, especially for high values of the final state invariant mass and the effect of missing higher orders is rapidly increasing with the final state invariant mass.

- When the cross-section $i j \rightarrow \mathrm{H}$ refers to an off-shell Higgs boson the choice of the QCD scales should be made

\footnotetext{
5 cf. http://personalpages.to.infn.it/ giampier/CPHTO.html.
}

according to the virtuality and not to a fixed value. Indeed, one must choose an infrared safe quantity fixed from the detectable final state, see Ref. [10]. Using the Higgs virtuality or the QCD scales has been advocated in Ref. [14]: the numerical impact is relevant, especially for high values of the invariant mass, the ratio static/dynamic scales being 1.05. The authors of Ref. [21] seem to agree on our choice [14].

- References $[3,21]$ consider the following scenario (onshell $\infty$-degeneracy): allow for a scaling of the Higgs couplings and of the total Higgs width defined by

$$
\begin{gathered}
\sigma_{i \rightarrow \mathrm{H} \rightarrow f}=(\sigma \cdot \mathrm{BR})=\frac{\sigma_{i}^{\mathrm{prod}} \Gamma_{f}}{\gamma_{\mathrm{H}}} \quad \sigma_{i \rightarrow \mathrm{H} \rightarrow f} \\
\propto \frac{g_{i}^{2} g_{f}^{2}}{\gamma_{\mathrm{H}}} \quad g_{i, f}=\xi g_{i, f}^{\mathrm{SM}}, \quad \gamma_{\mathrm{H}}=\xi^{4} \gamma_{\mathrm{H}}^{\mathrm{SM}}
\end{gathered}
$$

Looking for $\xi$-dependent effects in the highly off-shell region is an approach that raises sharp questions on the nature of the underlying extension of the SM; furthermore it does not take into account variations in the SM background and the signal strength in 41 , relative to the expectation for the SM Higgs boson, is measured by CMS to be $0.91_{-0.24}^{+0.30}$ [28] and by ATLAS to be $1.43_{-0.35}^{+0.40}$ [29]. We adopt the approach of Ref. [30] [in particular Eqs. (1-18)] which is based on the $\kappa$-language, allowing for a consistent "Higgs Effective Field Theory" (HEFT) interpretation, see Ref. [31]. Neglecting loop-induced vertices, we have

$$
\begin{aligned}
\frac{\Gamma_{\mathrm{gg}}}{\Gamma_{\mathrm{gg}}^{\mathrm{Sg}}\left(\mu_{\mathrm{H}}\right)} & =\frac{\kappa_{\mathrm{t}}^{2} \cdot \Gamma_{\mathrm{gg}}^{\mathrm{tt}}\left(\mu_{\mathrm{H}}\right)+\kappa_{\mathrm{b}}^{2} \cdot \Gamma_{\mathrm{gg}}^{\mathrm{bb}}\left(\mu_{\mathrm{H}}\right)+\kappa_{\mathrm{t}} \kappa_{\mathrm{b}} \cdot \Gamma_{\mathrm{gg}}^{\mathrm{tb}}\left(\mu_{\mathrm{H}}\right)}{\Gamma_{\mathrm{gg}}^{\mathrm{tt}}\left(\mu_{\mathrm{H}}\right)+\Gamma_{\mathrm{gg}}^{\mathrm{bb}}\left(\mu_{\mathrm{H}}\right)+\Gamma_{\mathrm{gg}}^{\mathrm{tb}}\left(\mu_{\mathrm{H}}\right)} \\
\sigma_{i \rightarrow \mathrm{H} \rightarrow f} & =\frac{\kappa_{i}^{2} \kappa_{f}^{2}}{\kappa_{\mathrm{H}}^{2}} \sigma_{i \rightarrow \mathrm{H} \rightarrow f}^{\mathrm{SM}}
\end{aligned}
$$

Remark The measure of off-shell effects can be interpreted as a constraint on $\gamma_{\mathrm{H}}$ only when we scale couplings and total width according to Eq. (32) to keep $\sigma_{\text {peak }}$ untouched, although its value is known with $15-20 \%$ accuracy.

Proposition 4.1 The generalization of Eq. (32) is an $\infty^{2}$ degeneracy, $\kappa_{i} \kappa_{f}=\kappa_{\mathrm{H}}$.

On the whole, we have a constraint in the multidimensional $\kappa$-space, since $\kappa_{\mathrm{g}}^{2}=\kappa_{\mathrm{g}}^{2}\left(\kappa_{\mathrm{t}}, \kappa_{\mathrm{b}}\right)$ and $\kappa_{\mathrm{H}}^{2}=\kappa_{\mathrm{H}}^{2}\left(\kappa_{j}, \forall j\right)$. Only on the assumption of degeneracy we can prove that off-shell effects "measure" $\kappa_{\mathrm{H}}$; a combination of on-shell effects (measuring $\kappa_{i} \kappa_{f} / \kappa_{\mathrm{H}}$ ) and off-shell effects [measuring $\kappa_{i} \kappa_{f}$, see Eq. (9)] gives information on $\kappa_{\mathrm{H}}$ without prejudices. Denoting by $S$ the signal and by I the interference and assuming that $\mathrm{I}_{\text {peak }}$ is negligible we have 


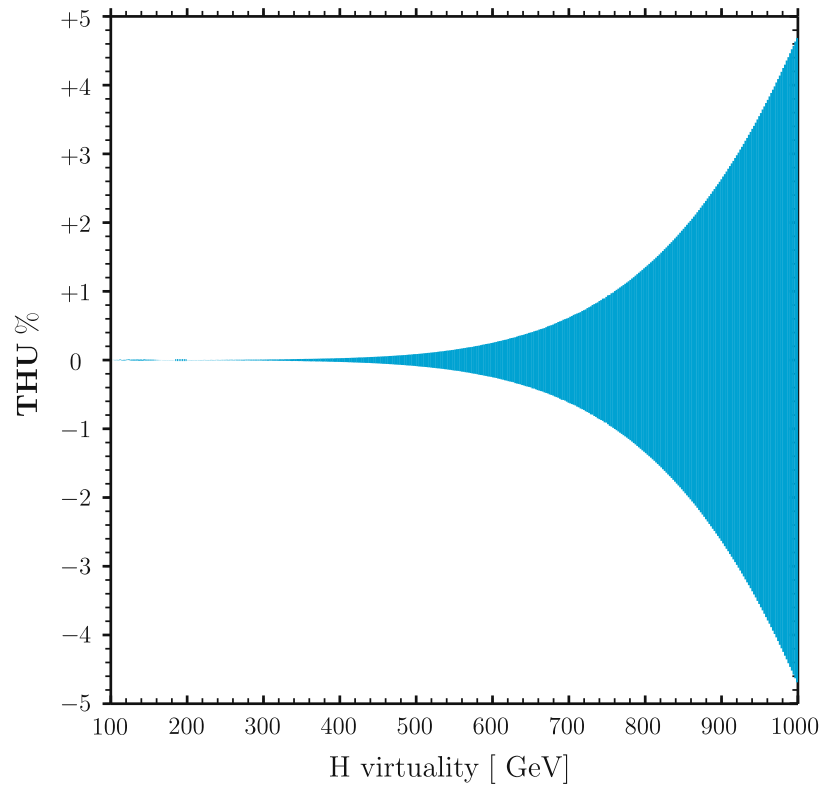

Fig. 6 Electroweak theoretical uncertainty for the signal lineshape at $\mu_{\mathrm{H}}=125.6 \mathrm{GeV}$

$\frac{\mathrm{S}_{\text {off }}}{\mathrm{S}_{\text {peak }}} \underset{\mathrm{H}}{\stackrel{2}{\mathrm{H}}}+\frac{\mathrm{I}_{\text {off }}}{\mathrm{S}_{\text {peak }}} \frac{\kappa_{\mathrm{H}}}{x_{i f}}, \quad x_{i f}=\frac{\kappa_{i} \kappa_{f}}{\kappa_{\mathrm{H}}}$

for the normalized $\mathrm{S}+\mathrm{I}$ off-shell cross section.

The background, e.g. gg $\rightarrow 41$, is also changed by the inclusion of $d=6$ operators and one cannot claim that New Physics is modifying only the signal. ${ }^{6}$

- The total systematic error is dominated by theoretical uncertainties, therefore one should never accept theoretical predictions that cannot provide uncertainty in a systematic way (i.e. providing an algorithm).

In Fig. 6 we consider the estimated theoretical uncertainty (THU) on the signal lineshape for a mass of $125.6 \mathrm{GeV}$. Note that PDF $+\alpha_{\mathrm{s}}$ and QCD scales uncertainties are not included. As expected for a light Higgs boson, the EW THU is sizable only for large values of the off-shell tail, reaching $\pm 4.7 \%$ at $1 \mathrm{TeV}$ (the algorithm is explained in Ref. [14]). To summarize the various sources of parametric (PU) and theoretical (THU) uncertainties, we have

\section{THU summary}

(1) $\mathrm{PDF}+\alpha_{\mathrm{s}}$; these have a Gaussian distribution;

(2) $\checkmark \mu_{\mathrm{R}}, \mu_{\mathrm{F}}$ (renormalization and factorization QCD scales) variations; they are the standard substitute for missing higher order uncertainty (MHOU) [32]; MHOU are better treated in a Bayesian context with a flat prior;

\footnotetext{
${ }^{6}$ Although one cannot disagree with von Neumann "With four parameters I can fit an elephant, and with five I can make him wiggle his trunk".
}

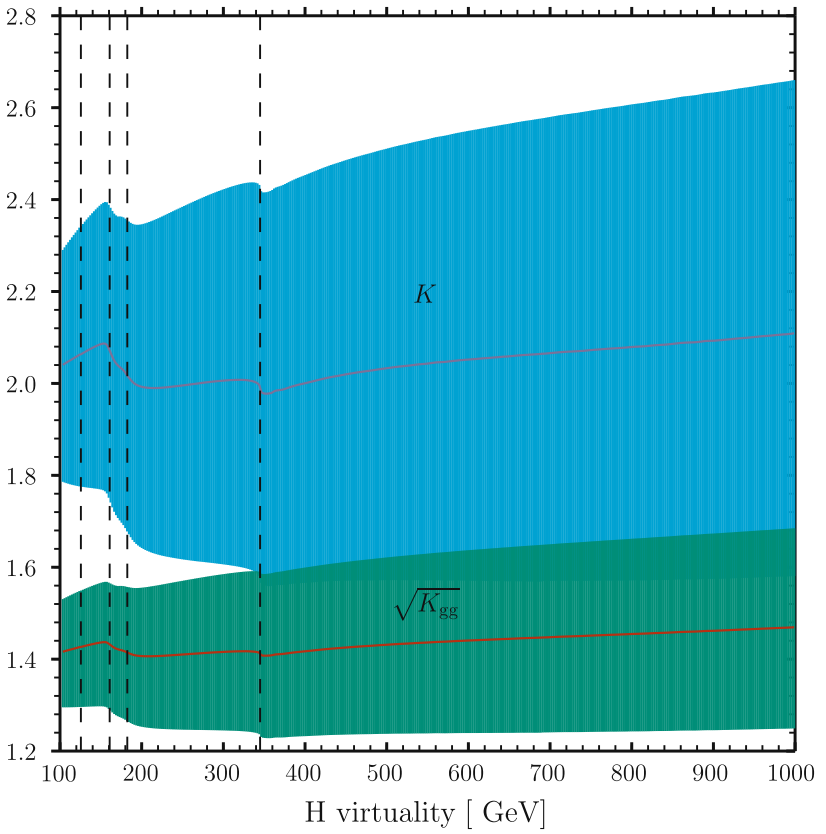

Fig. 7 Differential $K$-factors in Higgs production for $\mu_{\mathrm{H}}=$ $125.6 \mathrm{GeV}$. The central values correspond to $\mu_{\mathrm{R}}=\mu_{\mathrm{F}}=M_{\mathrm{f}} / 2$, where $M_{\mathrm{f}}$ is the Higgs virtuality. The bands give the THU simulated by varying QCD scales $\in\left[M_{\mathrm{f}} / 4, M_{\mathrm{f}}\right]$

(3) uncertainty on $\gamma_{\mathrm{H}}$ [Eq. (3)] due to missing higher orders, negligible for a light Higgs;

(4) $\checkmark$ uncertainty for $\Gamma_{\mathrm{H} \rightarrow \mathrm{F}}\left(M_{\mathrm{f}}\right)$ due to missing higher orders (mostly EW), especially for high values of the Higgs virtuality $M_{\mathrm{f}}$ (i.e. the invariant mass in $\mathrm{pp} \rightarrow \mathrm{H} \rightarrow \mathrm{f}+\mathrm{X}$ );

(5) $\checkmark$ uncertainty due to missing higher orders (mostly QCD) for the background

where $\checkmark$ means discussed in this note. When (2) is included one should remember the $\mathrm{N}^{3} \mathrm{LO}$ effect in gluon-gluon fusion (estimated $+17 \%$ in Ref. [33]) and and additional $+7 \%$ for an all-order estimate, see Ref. [32]. These numbers refer to the fully inclusive $\mathrm{K}$-factors. The effect of varying QCD scales, $\mu_{\mathrm{R}}=\mu_{\mathrm{F}} \in\left[M_{\mathrm{f}} / 4, M_{\mathrm{f}}\right]$ is shown in Fig. 7, for $\mathrm{K}$ and $\sqrt{\mathrm{K}_{\mathrm{gg}}}$.

Once again, it should be stressed that QCD scale variation is only a conventional simulation of the effect of missing higher orders. Taking Fig. 7 for its face value, we register a substantial reduction in the uncertainty when $\mathrm{K}$-factors are included. For instance, we find $[-12.1 \%,+11.0 \%]$ for the NNLO prediction around the peak, $[-10.9 \%,+9.9 \%]$ around $2 M_{\mathrm{Z}}$ and $[-9.7 \%,+6.6 \%]$ at $1 \mathrm{TeV}$. The corresponding LO prediction is $[-27.3 \%,+12.9 \%]$ around the peak, $[-29.5 \%,+32.1 \%]$ around $2 M_{Z}$ and $[-38 \%$, $+42 \%$ ] at $1 \mathrm{TeV}$. Note that $\mu_{\mathrm{R}}$ enters also in the values of $\alpha_{\mathrm{S}}$.

Admittedly, showing the effect of QCD scale variations on $\mathrm{K}$-factors is somewhat misleading but we have adopted this choice in view of the fact that, operatively speaking, the experimental analysis will generate bins in $M_{41}$ with a 


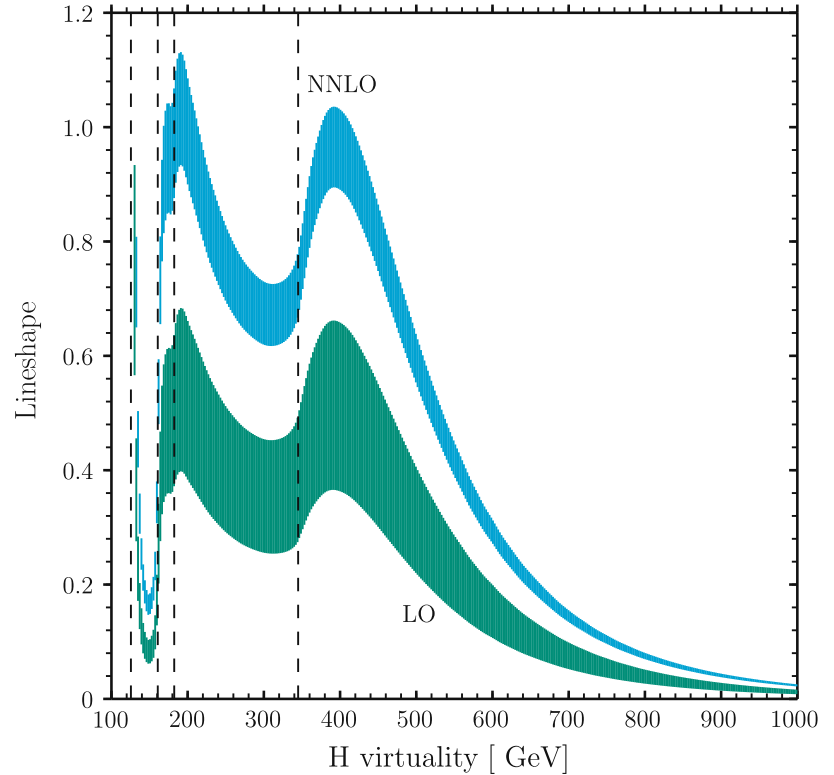

Fig. 8 (Camel) Lineshape for $\mu_{\mathrm{H}}=125.6 \mathrm{GeV}$. The central values correspond to $\mu_{\mathrm{R}}=\mu_{\mathrm{F}}=M_{\mathrm{f}} / 2$, where $M_{\mathrm{f}}$ is the Higgs virtuality. The bands give the THU simulated by varying QCD scales $\in\left[M_{\mathrm{f}} / 4, M_{\mathrm{f}}\right]$

LO MC and multiply the number of events in each bin by the corresponding $\mathrm{K}$-factor. Introducing $\mathrm{D}_{+}^{\mathrm{LO}}=\mathrm{D}^{\mathrm{LO}}\left(M_{\mathrm{f}} / 4\right)$ and $\mathrm{D}_{-}^{\mathrm{LO}}=\mathrm{D}^{\mathrm{LO}}\left(M_{\mathrm{f}}\right)$, where $\mathrm{D}^{\mathrm{LO}}$ is the $\mathrm{LO}$ distribution and $\mathrm{K}_{+}=\mathrm{K}\left(M_{\mathrm{f}} / 4\right)$ and $\mathrm{K}_{-}=\mathrm{K}\left(M_{\mathrm{f}}\right)$, where $\mathrm{K}=\mathrm{D}^{\mathrm{NNLO}} / \mathrm{D}^{\mathrm{LO}}$ is the $\mathrm{K}$-factor, the correct strategy is $\mathrm{K}_{ \pm} \mathrm{D}_{ \pm}^{\mathrm{LO}}$. When looking at Fig. 7 one should remember that the scale variation that increases (decreases) the distributions is the one decreasing (increasing) the $\mathrm{K}$-factor. The NNLO and LO (camelshaped) lineshapes, with QCD scale variations, are given in Fig. 8. The THU induced by QCD scale variation can be reduced by considering the (peak) normalized lineshape, as shown in Fig. 9. In other words the constraint on the Higgs intrinsic width should be derived by looking at the ratio

$\mathrm{R}_{\text {off }}^{41}=\frac{\mathrm{N}_{\text {off }}^{41}}{\mathrm{~N}_{\text {tot }}^{41}}, \quad \mathrm{~N}_{\mathrm{off}}^{41}=\mathrm{N}^{41}\left(M_{41}>M_{0}\right)$

as a function of $\gamma_{\mathrm{H}} / \gamma_{\mathrm{H}}{ }^{\mathrm{SM}}$, where $\mathrm{N}^{41}$ is the number of 4 leptons events. Since the $\mathrm{K}$-factor has a relatively small range of variation with the virtuality, the ratio in Eq. (35) is much less sensitive also to higher order terms.

An additional comment refers to Eqs. $(42,43)$ of Ref. [21], where $\gamma_{\mathrm{H}}=\gamma_{\mathrm{H}}{ }^{\mathrm{SM}}$ produces a negative number of events, a typical phenomenon that occurs with large and destructive interference effects when only signal + interference is considered. Unless the notion of negative events is introduced (background-subtracted number of events), the SM case cannot be included, as also shown in their Fig. 9, where only the portion $\gamma_{\mathrm{H}}>4.58(2.08) \gamma_{\mathrm{H}}{ }^{\mathrm{SM}}$ should be considered for $M_{41}>130(300) \mathrm{GeV}$, roughly a factor of 10 smaller than the estimated bounds. This clearly demonstrate the impor-

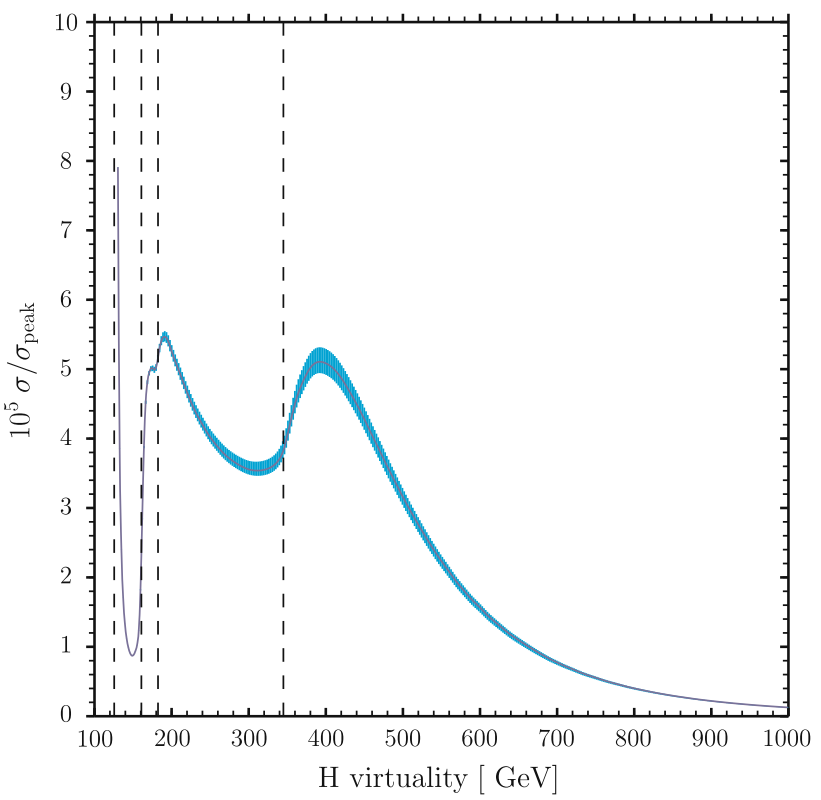

Fig. 9 Normalized NNLO lineshape for $\mu_{\mathrm{H}}=125.6 \mathrm{GeV}$. The central values correspond to $\mu_{\mathrm{R}}=\mu_{\mathrm{F}}=M_{\mathrm{f}} / 2$, where $M_{\mathrm{f}}$ is the Higgs virtuality. The bands give the THU simulated by varying QCD scales $\in\left[M_{\mathrm{f}} / 4, M_{\mathrm{f}}\right]$

tance of controlling THU on the interference, especially for improved limits on $\gamma_{\mathrm{H}}$.

\subsection{Improving THU for interference?}

One could argue that zero knowledge on the background $\mathrm{K}$ factor is a too conservative approach but it should be kept in mind that it's better to be with no one than to be with wrong one. Let us consider in details the process $i j \rightarrow \mathrm{F}$; the amplitude can be written as the sum of a resonant $(\mathrm{R})$ and a non-resonant (NR) part,

$A_{i j \rightarrow \mathrm{F}}=A_{i j \rightarrow \mathrm{H}} \frac{1}{s-s_{\mathrm{H}}} A_{\mathrm{H} \rightarrow \mathrm{F}}+A_{i j \rightarrow \mathrm{F}}^{\mathrm{NR}}$

We denote by LO the lowest order in perturbation theory where a process starts contributing and introduce $\mathrm{K}$-factors that include higher orders.

$\begin{aligned} A_{i j \rightarrow \mathrm{H}} & =\left(\mathrm{K}_{i j}^{\mathrm{p}}\right)^{1 / 2} A_{i j \rightarrow \mathrm{H}}^{\mathrm{LO}}, \quad A_{\mathrm{H} \rightarrow \mathrm{F}}=\left(\mathrm{K}_{\mathrm{F}}^{\mathrm{d}}\right)^{1 / 2} A_{\mathrm{H} \rightarrow \mathrm{F}}^{\mathrm{LO}}, \\ A_{i j \rightarrow \mathrm{F}}^{\mathrm{NR}} & =\left(\mathrm{K}_{i j \mathrm{~F}}^{\mathrm{b}}\right)^{1 / 2} A_{i j \rightarrow \mathrm{F}}^{\mathrm{NR}, \mathrm{LO}}\end{aligned}$

Furthermore, we introduce

$A_{i j \rightarrow \mathrm{F}}^{\mathrm{R}}=A_{i j \rightarrow \mathrm{H}} A_{\mathrm{H} \rightarrow \mathrm{F}}$

the interference becomes 


$$
\begin{aligned}
& \mathrm{I}=2\left[\mathrm{~K}_{i j}^{\mathrm{p}} \mathrm{K}_{\mathrm{F}}^{\mathrm{d}} \mathrm{K}_{i j \mathrm{~F}}^{\mathrm{b}}\right]^{1 / 2} \\
& \quad \times\left\{\operatorname{Re} \frac{A_{i j \rightarrow \mathrm{F}}^{\mathrm{R}, \mathrm{LO}}}{s-s_{\mathrm{H}}} \operatorname{Re} A_{i j \rightarrow \mathrm{F}}^{\mathrm{NR}, \mathrm{LO}}-\operatorname{Im} \frac{A_{i j \rightarrow \mathrm{F}}^{\mathrm{R}, \mathrm{LO}}}{s-s_{\mathrm{H}}} \operatorname{Im} A_{i j \rightarrow \mathrm{F}}^{\mathrm{NR}, \mathrm{LO}}\right\} \\
& \operatorname{Re} \frac{A_{i j \rightarrow \mathrm{F}}^{\mathrm{R}, \mathrm{F}}}{s-s_{\mathrm{H}}}=\frac{s-\mu_{\mathrm{H}}^{2}}{\left|s-s_{\mathrm{H}}\right|^{2}} \operatorname{Re} A_{i j \rightarrow \mathrm{F}}^{\mathrm{R}, \mathrm{LO}}+\frac{\mu_{\mathrm{H}} \gamma_{\mathrm{H}}}{\left|s-s_{\mathrm{H}}\right|^{2}} \operatorname{Im} A_{i j \rightarrow \mathrm{F}}^{\mathrm{R}, \mathrm{LO}} \\
& \operatorname{Im} \frac{A_{i j \rightarrow \mathrm{F}}^{\mathrm{R}, \mathrm{LO}}}{s-s_{\mathrm{H}}}=\frac{s-\mu_{\mathrm{H}}^{2}}{\left|s-s_{\mathrm{H}}\right|^{2}} \operatorname{Im} A_{i j \rightarrow \mathrm{F}}^{\mathrm{R}, \mathrm{LO}}-\frac{\mu_{\mathrm{H}} \gamma_{\mathrm{H}}}{\left|s-s_{\mathrm{H}}\right|^{2}} \operatorname{Re} A_{i j \rightarrow \mathrm{F}}^{\mathrm{R}, \mathrm{LO}}
\end{aligned}
$$

From Eq. (39) we see the main difference in the interference effects of a heavy Higgs boson w.r.t. the off-shell tail of a light Higgs boson. For the latter case $\gamma_{H}$ is completely negligible, whereas it gives sizable effects for the heavy Higgs boson case.

\subsubsection{The soft-knowledge scenario}

Neglecting PDF $+\alpha_{\mathrm{S}}$ uncertainties and those coming from missing higher orders, the major source of THU is due to the missing NLO interference. In Ref. [26] the effect of QCD corrections to the signal-background interference at the LHC has been studied for a heavy Higgs boson. A soft-collinear approximation to the NLO and NNLO corrections is constructed for the background process, which is exactly known only at LO. Its accuracy is estimated by constructing and comparing the same approximation to the exact result for the signal process, which is known up to NNLO, and the conclusion is that one can describe the signal-background interference to better than ten percent accuracy for large values of the Higgs virtuality. It is also shown that, in practice, a fairly good approximation to higher-order QCD corrections to the interference may be obtained by rescaling the known $\mathrm{LO}$ result by a $\mathrm{K}$-factor computed using the signal process.

The goodness of the approximation, when applied to the signal, remains fairly good down to $180 \mathrm{GeV}$ and rapidly deteriorates only below the $2 \mathrm{MZ}$-threshold; note that both $M_{41}>130 \mathrm{GeV}$ and $M_{41}>300 \mathrm{GeV}$ have been considered in the study of Ref. [21]. The exact result for the background is missing but the eikonal nature of the approximation should make it equally good, for signal as well as for background. ${ }^{7}$

This line of thought looks very promising, with a reduction of the corresponding THU (zero-knowledge scenario), although its extension from the heavy Higgs scenario to the light Higgs off-shell scenario has not been completely worked out. In a nutshell, one can write

\footnotetext{
${ }^{7}$ S. Forte, private communication.
}

$\sigma=\sigma^{\mathrm{LO}}+\sigma^{\mathrm{LO}} \frac{\alpha_{\mathrm{s}}}{2 \pi}[$ universal + process dependent + reg $]$

where "universal" (the + distribution) gives the bulk of the result while "process dependent" (the $\delta$ function) is known up to two loops for the signal but not for the background and "reg" is the regular part. A possible strategy would be to use for background the same "process dependent" coefficients and allow for their variation within some ad hoc factor. Assuming

$\mathrm{K}_{i j \mathrm{~F}}^{\mathrm{b} \text {, soft }}=\mathrm{K}_{i j}^{\mathrm{p}} \pm \Delta \mathrm{K}_{i j}^{ \pm}$

we could write

$$
\begin{aligned}
\mathrm{I} & =2 \mathrm{~K}_{i j}^{\mathrm{p}}\left(\mathrm{K}_{\mathrm{F}}^{\mathrm{d}}\right)^{1 / 2}\left[1 \pm \frac{\Delta \mathrm{K}_{i j}^{ \pm}}{\mathrm{K}_{i j}^{\mathrm{p}}}\right]^{1 / 2} \operatorname{Re} \frac{A_{i j \rightarrow \mathrm{F}}^{\mathrm{R}, \mathrm{LO}}}{s-s_{\mathrm{H}}}\left(A_{i j \rightarrow \mathrm{F}}^{\mathrm{NR} \mathrm{LO}}\right)^{*} \\
& =2 \mathrm{~K}_{i j}^{\mathrm{p}}\left(\mathrm{K}_{\mathrm{F}}^{\mathrm{d}}\right)^{1 / 2}\left[1 \pm \frac{\Delta \mathrm{K}_{i j}^{ \pm}}{\mathrm{K}_{i j}^{\mathrm{p}}}\right]^{1 / 2} \mathrm{I}^{\mathrm{LO}}
\end{aligned}
$$

In this scenario the subtraction of the background cannot be performed at LO. It is worth noting that simultaneous inclusion of higher order corrections for Higgs production (NNLO) and Higgs decay (NLO) is a three-loop effect that is not balanced even with the introduction of the eikonal QCD $\mathrm{K}$ factor for the background; three loop mixed EW-QCD corrections are still missing, even at some approximate level. Note that $\mathrm{K}_{41}^{\mathrm{d}}$ can be obtained by running PROPHECY4F [34] in $\mathrm{LO} / \mathrm{NLO}$ modes.

\subsection{Background-subtracted lineshape}

In Fig. 10 we present our results for $\sigma^{\mathrm{S}+\mathrm{I}}$ for the $\mathrm{ZZ} \rightarrow 4 \mathrm{e}$ final state. The pseudo-observable $\sigma^{\mathrm{S}+\mathrm{I}}$ that includes only signal and interference (not constrained to be positive) is now a standard in the experimental analysis.

The blue curve in Fig. 10 gives the intermediate option for including the interference and the cyan band the associated THU between additive and multiplicative options. Multiplicative option is the green curve. Red curves give the THU due to QCD scale variation for the intermediate option (QCD scales $\in\left[M_{\mathrm{f}} / 4, M_{\mathrm{f}}\right]$, where $M_{\mathrm{f}}=M_{4 \mathrm{e}}$ is the Higgs virtuality). A cut $p_{\mathrm{T}}^{\mathrm{Z}}>0.25 M_{4 \mathrm{e}}$ has been applied. The figure shows how a $\mathrm{S}$ (camel-shaped) distributions transforms into a $\mathrm{S}+\mathrm{I}$ (square-root-shaped) distribution.

Remark Of course, one could adopt the soft-knowledge recipe, in which case the result is given by the green curve in Fig. 10; provisionally, one could assume a $\pm 10 \%$ uncertainty, extrapolating the estimate made for the high-mass study in Ref. [26]. Background subtraction should be performed accordingly [ $\mathrm{K}_{i j \mathrm{~F}}^{\mathrm{b}}$ of Eq. (37)]. 


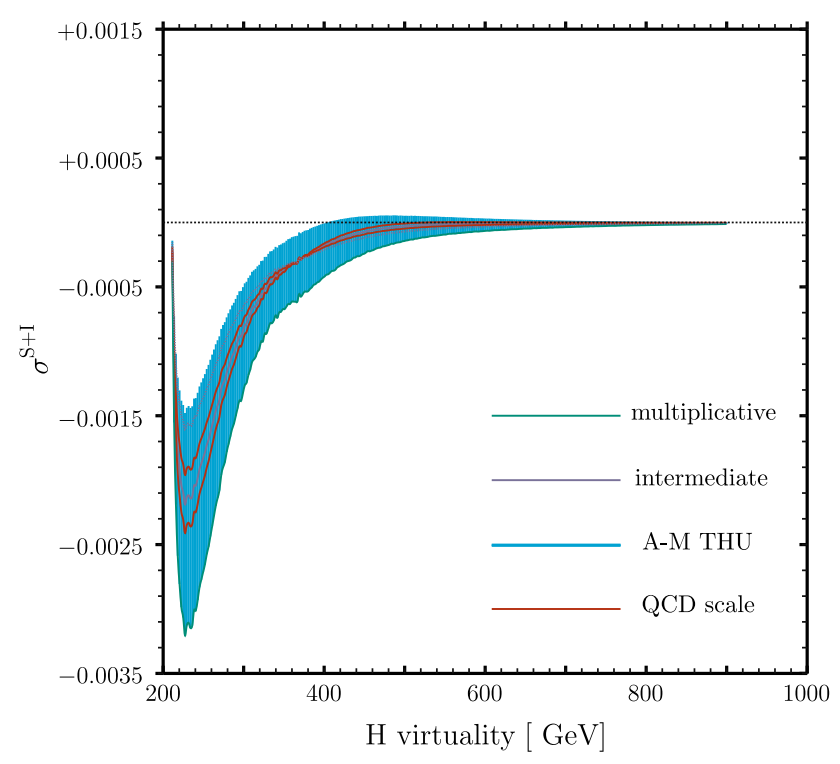

Fig. $10 \sigma^{\mathrm{S}+\mathrm{I}}$ for $4 \mathrm{e}$ final state. The blue curve gives the intermediate option and the cyan band the associated THU between additive and multiplicative options. Multiplicative option is the green curve. Red curves give the THU due to QCD scale variation for the intermediate option (QCD scales $\in\left[M_{\mathrm{f}} / 4, M_{\mathrm{f}}\right]$, where $M_{\mathrm{f}}=M_{4 \mathrm{e}}$ is the Higgs virtuality). A cut $p_{\mathrm{T}}^{\mathrm{Z}}>0.25 M_{4 \mathrm{e}}$ has been applied. If one adopts the softknowledge recipe, the result is given by the green curve; provisionally, one could assume a $\pm 10 \%$ uncertainty, extrapolating the estimate made for the high-mass study in Ref. [26]

It is worth introducing few auxiliary quantities [15]: the minimum and the half-minima of $\sigma^{\mathrm{S}+\mathrm{I}}$ : given

$\mathrm{D}\left(M_{41}\right)=\frac{d}{d M_{41}^{2}} \sigma^{\mathrm{S}+\mathrm{I}}$

we define

$$
\begin{gathered}
\mathrm{D}_{1}=\mathrm{D}\left(M_{1}\right)=\min \mathrm{D}\left(M_{41}\right), \\
\mathrm{D}_{1 / 2}^{ \pm}=\mathrm{D}\left(M_{1 / 2}^{ \pm}\right)=\frac{1}{2} \mathrm{D}\left(M_{1}\right)
\end{gathered}
$$

As observed in Ref. [15], THU is tiny on $M_{1}$ and moderately larger for $M_{1 / 2}^{ \pm}$.

Remark Alternatively, and taking into account the indication of Ref. [26] we could proceed as follows: ${ }^{8}$ we can try to turn our three measures of the lineshape into a continuous estimate in each bin; there is a technique, called "vertical morphing" [35], that introduces a "morphing" parameter $f$ which is nominally zero and has some uncertainty. If we define

$$
\begin{aligned}
\mathrm{D}^{0} & =\frac{d \sigma^{\mathrm{S}+\mathrm{I}}}{d M_{41}^{2}}, \quad \text { option } \mathrm{I}, \quad \mathrm{D}^{+}=\max _{\mathrm{A}, \mathrm{M}} \mathrm{D}, \\
\mathrm{D}^{-} & =\min _{\mathrm{A}, \mathrm{M}} \mathrm{D}
\end{aligned}
$$

\footnotetext{
${ }^{8}$ I gratefully acknowledge the suggestion by S. Bolognesi.
}

the simplest "vertical morphing" replaces

$\mathrm{D}^{0} \rightarrow \mathrm{D}^{0}+\frac{f}{2}\left(\mathrm{D}^{+}-\mathrm{D}^{-}\right)$

Of course, the whole idea depends on the choice of the distribution for $f$, usually Gaussian which is not necessarily our case; instead, one would prefer to maintain, as much as possible, the indication from the soft-knowledge scenario (in a Bayesian sense). Therefore, we define two curves

$$
\begin{gathered}
\mathrm{D}_{-}\left(\lambda, M_{41}\right)=\lambda \mathrm{D}_{\mathrm{M}}\left(M_{41}\right)+(1-\lambda) \mathrm{D}_{\mathrm{I}}\left(M_{41}\right) \\
\mathrm{D}_{+}\left(\lambda, M_{41}\right)=\lambda \mathrm{D}_{\mathrm{I}}\left(M_{41}\right)+(1-\lambda) \mathrm{D}_{\mathrm{A}}\left(M_{41}\right)
\end{gathered}
$$

We assume that the parameter $\lambda$, with $0 \leq \lambda \leq 1$, has a flat distribution. We will have $\mathrm{D}_{-}<\mathrm{D}_{\mathrm{I}}<\mathrm{D}_{+}$and a value for $\lambda$ close to one (e.g. 0.9) gives less weight to the additive option, highly disfavored by the eikonal approximation. The corresponding THU band will be labelled by $\operatorname{VM}(\lambda)$.

Consider $\mathrm{D}_{1}$ of Eq. (44): we have $M_{1}=233.9 \mathrm{GeV}$ and the THU band corresponding to the full variation between Aoption and M-option is $0.00171 \mathrm{fb}$, equivalent to $\mathrm{a} \pm 39.9 \%$. If we select $\lambda=0.9$ in Eq. (47) the difference $\mathrm{D}_{-}$ $\mathrm{D}_{+}$reduces the uncertainty to $0.00098 \mathrm{fb}$, equivalent to $\pm 22.8 \%$. The destructive effect of the interference shows how challenging will be to put more stringent bounds on $\gamma_{\mathrm{H}}$ when $\gamma_{\mathrm{H}} \rightarrow \gamma_{\mathrm{H}}{ }^{\mathrm{SM}}$. The off-shell effects are an ideal place where to look for "large" deviations from the SM (from $\gamma_{\mathrm{H}}{ }^{\mathrm{SM}}$ ) where, however, large scaling of the Higgs couplings raise severe questions on the structure of underlying BSM theory.

Definition There is an additional variable that we should consider:

$\mathrm{R}^{\mathrm{S}+\mathrm{I}}\left(M_{1}, M_{2}\right)=\frac{\sigma^{\mathrm{S}+\mathrm{I}}\left(M_{41}>M_{1}\right)}{\sigma^{\mathrm{S}+\mathrm{I}}\left(M_{41}>M_{2}\right)}$

For instance, integrate $d \sigma^{\mathrm{S}+\mathrm{I}} / d M_{41}^{2}$ over bins of $2.25 \mathrm{GeV}$ for $M_{41}>212 \mathrm{GeV}$ and obtain $\sigma^{\mathrm{S}+\mathrm{I}}(i)$. Next, consider the ratio $\mathrm{R}^{\mathrm{S}+\mathrm{I}}(i)=\sigma^{\mathrm{S}+\mathrm{I}}(i) / \sigma^{\mathrm{S}+\mathrm{I}}(1)$ which is shown in Fig. 11 where the THU band is given by $\operatorname{VM}(0.9)$. To give an example the THU corresponding to the bin of $300 \mathrm{GeV}$ is $14.9 \%$. THU associated with QCD scale variations is given by the two dashed lines.

\section{Conclusions}

The successful search for the on-shell Higgs-like boson has put little emphasis on the potential of the off-shell events; the attitude was "the issue of the Higgs off-shellness is very interesting but it is not relevant for low Higgs masses" and "for SM Higgs below $200 \mathrm{GeV}$, the natural width (mostly for MSSM as well) is much below the experimental resolution. 


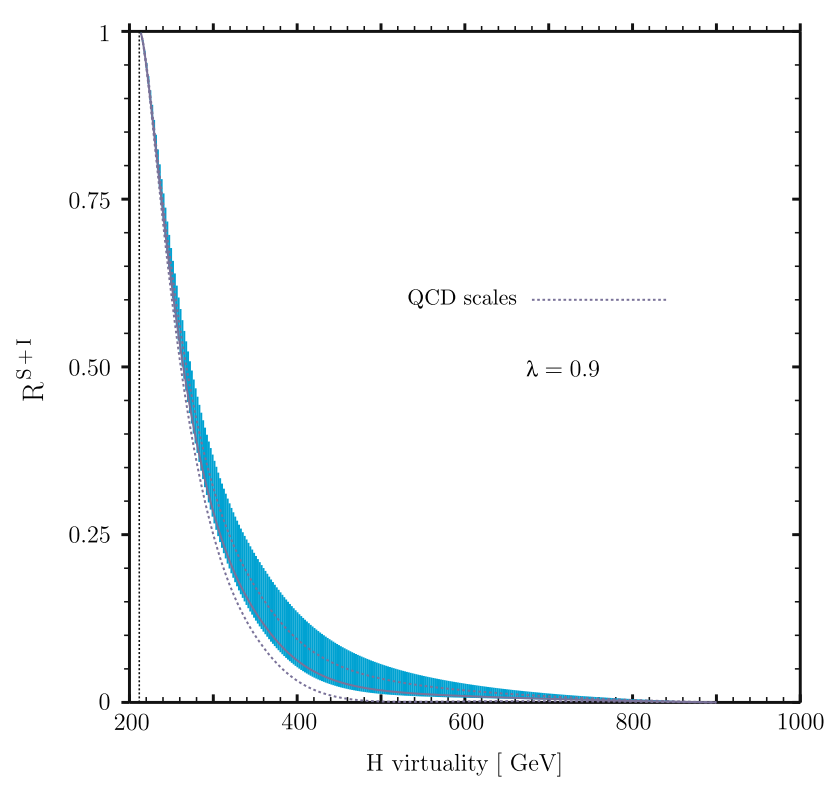

Fig. 11 The ratio $\mathrm{R}^{\mathrm{S}+\mathrm{I}}(i)=\sigma^{\mathrm{S}+\mathrm{I}}(i) / \sigma^{\mathrm{S}+\mathrm{I}}(1)$, Eq. (48), where $\sigma^{\mathrm{S}+\mathrm{I}}(i)$ is obtained by integrating $d \sigma^{\mathrm{S}+\mathrm{I}} / d M_{4 \mathrm{l}}^{2}$ over bins of $2.25 \mathrm{GeV}$ for $M_{41}>212 \mathrm{GeV}$. The parameter $\lambda$ is defined in Eq. (47). Dashed lines give the QCD scale variation $\left(\mathrm{QCD}\right.$ scales $\in\left[M_{\mathrm{f}} / 4, M_{\mathrm{f}}\right]$, where $M_{\mathrm{f}}=M_{4 \mathrm{e}}$ is the Higgs virtuality). A cut $p_{\mathrm{T}}^{\mathrm{Z}}>0.25 M_{4 \mathrm{e}}$ has been applied

We have therefore never cared about it for light Higgs. Just produce on-shell Higgs and let them decay in MC"; luckily the panorama is changing.

In 2012, it was demonstrated that, with few assumptions and using events with pairs of $\mathrm{Z}$ particles, the high invariant mass tail can be used to constrain the Higgs width. One can also extract an upper limit on the Higgs width at the price of assuming that its couplings to the known particles are given by the Standard Model, yet allowing new particles to affect the width: the LHC is becoming a precision instrument even in the Higgs sector.

It is clear that one can't do much without a MC, therefore the analysis should be based on some LO MC, or some other. However, more inclusive NLO (or even NNLO) calculations show that the LO predictions can be far away, which means that re-weighting can be a better approximation, as long as it is accompanied by an algorithmic formulation of the associated theoretical uncertainty. The latter is (almost) dominating the total systematic error and precision Higgs physics requires control of both systematics, not only the experimental one. Very often THU is nothing more than educated guesswork but a workable falsehood is more useful than a complex incomprehensible truth. In other words, closeness to the whole truth is in part a matter of degree of informativeness of a proposition.

Acknowledgments Work supported by MIUR under contract 2001023713_006 and by UniTo - Compagnia di San Paolo under contract ORTO11TPXK.
Open Access This article is distributed under the terms of the Creative Commons Attribution License which permits any use, distribution, and reproduction in any medium, provided the original author(s) and the source are credited.

Funded by $\mathrm{SCOAP}^{3}$ / License Version CC BY 4.0.

\section{Appendix A: Analytic separation of off-shell effects}

The effect of non-SM Higgs couplings on $\sigma^{\mathrm{S}+\mathrm{I}}$ can be computed under the assumption $\gamma_{\mathrm{H}} \ll \mu_{\mathrm{H}}$. Consider the following integral:

$F_{i j}=\int_{z_{0}}^{1} d z \int_{z}^{1} \frac{d v}{v} \mathscr{L}_{i j}(v)\left|f_{i j}(s, z, v)\right|^{2}$

where the amplitude $f$ is

$f_{i j}(s, z, v)=\frac{A_{i j}(z, v)}{z s-s_{\mathrm{H}}}+B_{i j}(z, v)$

and where $i j$ denotes gg or $\overline{\mathrm{q}} \mathrm{q}$. For the process $i j \rightarrow \mathrm{F}$ we have $A_{i j} \propto g_{i j \mathrm{H}} g_{\mathrm{HF}}$ and $A_{i J}$ is related to $\sigma_{i j \rightarrow \mathrm{H}}, \Gamma_{\mathrm{H} \rightarrow \mathrm{F}}$ by Eq. (9). Simple expressions can be derived if we neglect the dependence of $A_{i j}, B_{i j}$ on the kinematic variables (but both contain thresholds). Using instead the results of Ref. [11] $\left(\gamma_{\mathrm{H}} \ll \mu_{\mathrm{H}}\right)$ we obtain

$$
\begin{aligned}
\frac{1}{\left|z s-s_{\mathrm{H}}\right|^{2}} & =\frac{\pi}{\mu_{\mathrm{H}} \gamma_{\mathrm{H}}} \delta\left(z s-\mu_{\mathrm{H}}^{2}\right)+\mathrm{PV}\left[\frac{1}{\left(z s-\mu_{\mathrm{H}}^{2}\right)^{2}}\right], \\
\mathrm{PV}\left(\frac{1}{z^{n}}\right) & =\frac{(-1)^{n-1}}{(n-1) !} \frac{d^{n}}{d z^{n}} \ln (|z|)
\end{aligned}
$$

we introduce $\hat{\mu}_{\mathrm{H}}^{2}=\mu_{\mathrm{H}}^{2} / s, z_{\mathrm{H}}=z+\hat{\mu}_{\mathrm{H}}^{2}$ and

$$
\begin{aligned}
& \mathscr{F}_{i j}^{\mathrm{S}}(z, v)=\left|A_{i j}(z, v)\right|^{2}, \\
& \mathscr{F}_{i j}^{\mathrm{B}}(z, v)=\left|\left(z s-s_{\mathrm{H}}\right) B_{i j}(z, v)\right|^{2}, \\
& \mathscr{F}_{i j}^{\mathrm{I}}(z, v)=\left(z s-s_{\mathrm{H}}{ }^{*}\right) A_{i j}(z, v) B_{i j}^{*}(z, v)
\end{aligned}
$$

obtaining the following result for the off-shell part of the integral in Eq. (49) $\left(z_{0}>\hat{\mu}_{\mathrm{H}}^{2}\right)$

$$
\begin{aligned}
F_{\text {off }}= & -\frac{1}{s^{2}} \int_{z_{0}}^{1} \frac{d v}{v} \mathscr{L}_{i j}(v) \int_{z_{0}-\hat{\mu}_{\mathrm{H}}^{2}}^{v-\hat{\mu}_{\mathrm{H}}^{2}} d z\left[\mathscr{F}_{i j}^{\mathrm{S}}\left(z_{\mathrm{H}}, v\right)\right. \\
& \left.+\mathscr{F}_{i j}^{\mathrm{B}}\left(z_{\mathrm{H}}, v\right)+2 \operatorname{Re} \mathscr{F}_{i j}^{\mathrm{I}}\left(z_{\mathrm{H}}, v\right)\right] \frac{d^{2}}{d z^{2}} \ln z
\end{aligned}
$$


Since [36]

$$
\begin{aligned}
\int_{a}^{b} d z g(z) \frac{d^{2}}{d z^{2}} \ln z= & {\left.\left[\frac{g(z)}{z}-g^{\prime}(z) \ln z\right]\right|_{a} ^{b} } \\
& +\int_{a}^{b} d z g^{\prime \prime}(z) \ln z
\end{aligned}
$$

we derive that the exact behavior of $F_{\text {off }}$ is controlled by the amplitude and by its first two derivatives. The form factors $\mathscr{F}^{l}$ admit a formal expansion in $\alpha_{\mathrm{s}}$ given by

$$
\begin{aligned}
\mathscr{F}_{i j}^{l}(z, v)= & \mathscr{F}_{i}^{l, 0}(z) \delta\left(1-\frac{z}{v}\right) \\
& +\sum_{n=1}^{\infty}\left(\frac{\alpha_{\mathrm{s}}\left(\mu_{\mathrm{R}}\right)}{\pi}\right)^{n} \mathscr{F}_{i j}^{l, n}(z, v)
\end{aligned}
$$

where we have considered QCD corrections but not the EW.

\section{References}

1. N. Kauer, G. Passarino, Inadequacy of zero-width approximation for a light higgs boson signal. JHEP 1208, 116 (2012). arXiv:1206.4803 [hep-ph]

2. N. Kauer, Inadequacy of zero-width approximation for a light higgs boson signal. Mod. Phys. Lett. A 28, 1330015 (2013). arXiv:1305.2092 [hep-ph]

3. F. Caola, K. Melnikov, Constraining the higgs boson width with zz production at the lhc. Phys. Rev. D 88, 054024 (2013). arXiv:1307.4935 [hep-ph]

4. J.D. Jackson, D. Scharre, Initial state radiative and resolution corrections and resonance parameters in e+ e- annihilation. Nucl. Instrum. Meth. 128, 13 (1975)

5. J.P. Alexander, G. Bonvicini, P.S. Drell, R. Frey, Radiative corrections to the $\mathrm{Z}^{0}$ resonance. Phys. Rev. D 37, 56-70 (1988)

6. D.Y. Bardin, G. Passarino, The Standard Model in the Making: Precision Study of the Electroweak Interactions (Clarendon Press, Oxford, 1999)

7. G. Passarino, Higgs pseudo-observables. Nucl. Phys. Proc. Suppl. 205-206, 16-19 (2010)

8. G. Passarino, C. Sturm, S. Uccirati, Higgs pseudo-observables, second riemann sheet and all that. Nucl. Phys. B 834, 77-115 (2010). arXiv: 1001.3360 [hep-ph]

9. M. Spira, A. Djouadi, D. Graudenz, P.M. Zerwas, Higgs boson production at the lhc. Nucl. Phys. B 453, 17-82 (1995). hep-ph/9504378

10. J.C. Collins, D.E. Soper, G.F. Sterman, Factorization of hard processes in QCD. Adv. Ser. Direct. High Energy Phys. 5, 1-91 (1988). arXiv:hep-ph/0409313 [hep-ph]. To be publ. in 'Perturbative QCD' (A.H. Mueller, ed.) (World Scientific Publ., 1989)

11. F.V. Tkachov, On the structure of systematic perturbation theory with unstable fields. arXiv:hep-ph/0001220 [hep-ph]

12. J.M. Campbell, R.K. Ellis, C. Williams, Gluon-gluon contributions to $\mathrm{w}^{-} \mathrm{w}^{+}$production and higgs interference effects. JHEP 1110, 005 (2011). arXiv:1107.5569 [hep-ph]

13. S. Actis, G. Passarino, C. Sturm, S. Uccirati, Nlo electroweak corrections to higgs boson production at hadron colliders. Phys. Lett. B 670, 12-17 (2008). arXiv:0809.1301 [hep-ph]

14. S. Goria, G. Passarino, D. Rosco, The higgs boson lineshape. Nucl. Phys. B 864, 530-579 (2012). arXiv:1112.5517 [hep-ph]
15. G. Passarino, Higgs interference effects in $g g \rightarrow z z$ and their uncertainty. JHEP 1208, 146 (2012). arXiv:1206.3824 [hep-ph]

16. S.P. Martin, Shift in the lhc higgs diphoton mass peak from interference with background. Phys. Rev. D 86, 073016 (2012). arXiv: 1208.1533 [hep-ph]

17. S.P. Martin, Interference of Higgs diphoton signal and background in production with a jet at the LHC. Phys. Rev. D 88(1), 013004 (2013). arXiv:1303.3342 [hep-ph]

18. L.J. Dixon, M.S. Siu, Resonance-continuum interference in the diphoton higgs signal at the lhc. Phys. Rev. Lett. 90, 252001 (2003). arXiv:hep-ph/0302233

19. L.J. Dixon, Y. Li, Bounding the higgs boson width through interferometry. Phys. Rev. Lett. 111, 111802 (2013). arXiv:1305.3854 [hep-ph]

20. D. de Florian, N. Fidanza, R. Hernández-Pinto, J. Mazzitelli, Y. Rotstein Habarnau et al., A complete $\mathscr{O}\left(\alpha_{\mathrm{s}}^{2}\right)$ calculation of the signal-background interference for the Higgs diphoton decay channel. Eur. Phys. J. C 73, 2387 (2013). arXiv:1303.1397 [hep-ph]

21. J.M. Campbell, R.K. Ellis, C. Williams, Bounding the Higgs width at the LHC using full analytic results for $\mathrm{gg} \rightarrow 2 \mathrm{e} 2 \mu$. arXiv:1311.3589 [hep-ph]

22. N. Kauer, Interference effects for $\mathrm{H} \rightarrow \mathrm{WW} / \mathrm{ZZ} \rightarrow 1 \bar{\nu}_{1} \bar{l} \nu_{1}$ searches in gluon fusion at the LHC. arXiv:1310.7011 [hep-ph]

23. J.M. Campbell, W.T. Giele, C. Williams, Event-by-event weighting at next-to-leading order. arXiv:1311.5811 [hep-ph]

24. J.M. Campbell, R.K. Ellis, C. Williams, Bounding the Higgs width at the LHC: complementary results from $\mathrm{H} \rightarrow$ WW. arXiv:1312.1628 [hep-ph]

25. I. Anderson, S. Bolognesi, F. Caola, Y. Gao, A.V. Gritsan et al., Constraining anomalous hvv interactions at proton and lepton colliders. Phys. Rev. D 89, 035007 (2014). arXiv:1309.4819 [hep-ph]

26. M. Bonvini, F. Caola, S. Forte, K. Melnikov, G. Ridolfi, Signalbackground interference effects for $g g \rightarrow h \rightarrow w^{+} w^{-}$beyond leading order. Phys. Rev. D 88, 034032 (2013). arXiv:1304.3053 [hep-ph]

27. G. Davatz, F. Stockli, C. Anastasiou, G. Dissertori, M. Dittmar et al., Combining monte carlo generators with next-to-next-to-leading order calculations: event reweighting for higgs boson production at the lhc. JHEP 0607, 037 (2006). arXiv:hep-ph/0604077 [hep-ph]

28. CMS Collaboration Collaboration, Properties of the Higgs-like boson in the decay $\mathrm{H}$ to $\mathrm{ZZ}$ to 41 in pp collisions at sqrt $\mathrm{s}=7$ and $8 \mathrm{TeV}$

29. ATLAS Collaboration Collaboration, G. Aad et al., Measurements of Higgs boson production and couplings in diboson final states with the ATLAS detector at the LHC. Phys. Lett. B 726, 88-119 (2013). arXiv:1307.1427 [hep-ex]

30. LHC Higgs Cross Section Working Group Collaboration, A. David et al., LHC HXSWG interim recommendations to explore the coupling structure of a Higgs-like particle. arXiv:1209.0040 [hep-ph]

31. G. Passarino, Nlo inspired effective lagrangians for higgs physics. Nucl. Phys. B 868, 416-458 (2013). arXiv:1209.5538 [hep-ph]

32. A. David, G. Passarino, How well can we guess theoretical uncertainties? Phys. Lett. B 726, 266-272 (2013). arXiv:1307.1843 [hep-ph]

33. R.D. Ball, M. Bonvini, S. Forte, S. Marzani, G. Ridolfi, Higgs production in gluon fusion beyond nnlo. Nucl. Phys. B 874, 746772 (2013). arXiv:1303.3590 [hep-ph]

34. A. Bredenstein, A. Denner, S. Dittmaier, M. Weber, Precision calculations for $\mathrm{H} \rightarrow \mathrm{WW} / \mathrm{ZZ} \rightarrow 4 \mathrm{l}$; with PROPHECY4f. arXiv:0708.4123 [hep-ph]

35. J. Conway, Incorporating nuisance parameters in likelihoods for multisource spectra. arXiv:1103.0354 [physics.data-an]

36. M. Nekrasov, Integral in the sense of principal value as a distribution over parameters of integration, arXiv:math-ph/ 0303024 [math-ph] 